\title{
Performance Analysis of Time-Delay Estimation Systems
}

\author{
by \\ Ehud Weinstein \\ Woods Hole Oceanographic Institution \\ Woods Hole, Massachusetts 02543
}

August 1984

Technical Report

This technical report was prepared for the Naval Underwater Systems Center, New London, Connecticut, under Contract N00140-83-C-KA35.

Reproduction in whole or in part is permitted for any purpose of the United States Government. This report should be cited as:

Woods Hole Oceanog. Inst. Tech. Rept. WHOI-84-32.

Approved for public release; distribution unlimited.

Approved for Distribution:

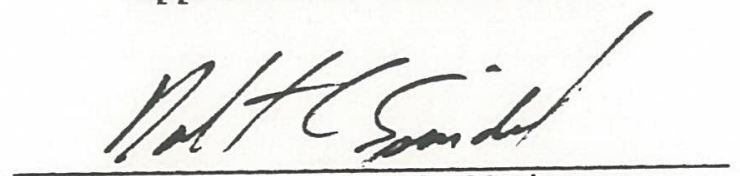

Robert C. Spindel, Chairman

Department of Ocean Engineering 


\section{TABLE OF CONTENTS}

Page

ABSTRACT

I. INTRODUCTION

A. Ambiguity Phenomena in Time Delay Estimation 1

B. Problem Formulation and Assumptions 3

C. The Modified Ziv-Zakai Lower Bound 5

II. BASEBAND SYSTEMS 6

$\begin{array}{ll}\text { III. BANDPASS SYSTEMS } & 10\end{array}$

IV. CONCLUSIONS

$\begin{array}{ll}\text { Acknowledgements } & 17\end{array}$

APPENDIX A. Lower Bounds on $\mathrm{Pe}_{\mathrm{e}}(\mathrm{x}) \quad 18$

APPENDIX B. Analysis of the Modified ZZLB for Baseband Signals

APPENDIX C. Analysis of the Modified ZZLB for Bandpass Signals 
ABSTRACT

This is the second part of a study which deals with the problem of passive time delay estimation. The focus here is on systems employing wideband signals and/or arrays of very widely separated receivers. A modified (improved) version of the Ziv-Zakai lower bound (ZZLB) is used to analyze the effect of additive noise and signal ambiguities on the attainable mean square estimation errors. When the lower bound is plotted as a function of signal-to-noise ratio (SNR) one observes two distinct threshold phenomena dividing the SNR domain into three disjointed segments: at high SNR the lower bound coincides with the Cramer-Rao lower bound (CRLB). This is the ambiguity-free mode of operation where differential delay estimation is subject only to local errors. At moderate SNR (between the two thresholds), the lower bound exceeds the CRLB by a factor of $12\left(\omega_{0} / \mathrm{W}\right)^{2}$ where $\omega_{0}$ and $W$ are, respectively, the center frequency and signal bandwidth. In this region the ambiguities in the received signal phases cannot be resolved, however a useful estimate of the differential delay can still be obtained using the received signal envelopes. At low SNR, the lower bound approaches a constant level depending only on the variance of the a-priori search domain of the unknown delay parameter. In this region signal observations are subject to envelope ambiguities as well, thus essentially useless for the delay estimation. 


\section{INTRODUCTION}

\section{A. Ambiguity Phenomena in Time Delay Estimation}

Estimation of the time difference of arrival of a noise-like random signal observed at two or more spatially separated receivers is a problem of considerable practical interest in many disciplines such as underwater acoustics, geophysics and radio astronomy, to mention a few. Consequently, numerous procedures have been proposed for passive time-delay estimation (e.g. $[1]-[7])$. In most of the systems which have been analyzed the Cramer-Rao inequality has been used to set a lower bound on the attainable mean square estimation errors. Its use was justified by invoking a well known theorem in statistics asserting that the maximum likelihood (ML) estimator is asymptotically unbiased and that its error variance approaches the Cramer-Rao lower bound (CRLB) arbitrarily close for sufficiently long observation times. There remains the question: How long is "long enough"? Clearly, the observation time $T$ must be 1 arge compared with the correlation time (inverse bandwidth) of signal and noise $(W T / 2 \pi \gg 1)$, a condition which presents very little difficulty in practice. However, if one examines the problem more closely, one finds a second condition: the ML estimator (which approaches the CRLB asymptotically) must not be subject to ambiguities.

Perhaps the most common setting in which this difficulty occurs is in delay estimation using very narrowband signals. Consider the extreme case of observations at only two receivers so that only one differential delay can be estimated. The ML estimate of that delay cross correlates the received signals, averages for time $T$, and obtaines the desired delay from the peak of 
the cross correlation function. In the narrowband case, the differential delay causes essentially to a phase shift between the received signals and thus generating a formidable ambiguity problem. This is illustrated in Figure 1. The cross correlation output peaks at $\Delta \tau$, the true differential delay, but is quasi-periodic with a period of $2 \pi / \omega_{0}$ where $\omega_{0}$ is the center frequency of the signal. To come close to the CRLB one must be able to distinguish unambiguously between adjacent peaks of the correlation function. If the signal bandwidth is only a small fraction of its center frequency (i.e., $\left.W / \omega_{0} \ll 1\right)$, adjacent peaks have very nearly equal height and identification of the largest one will require either very large SNR or exceedingly long observation times. In many important practical situations, therefore, the attainable m.s.e. may be very drastically inferior to that predicted by the CRLB.

In [8], a new lower bound, based on a modified (improved) version of the Ziv-Zakai lower bound (ZZLB), is developed to analyze the attainable mean square error (m.s.e.) in delay estimation schemes. The resulting lower bound is then applied to investigate the effect of ambiguity on delay estimation using narrowband signals [9]. When the lower bound is plotted as a function of SNR, one observes a strong threshold phenomenon. Above a critical SNR the lower bound approaches the CRLB. In this region the indicated phase ambiguities in the differential delay observations can essentially be resolved. Below the threshold, the lower bound exceeds the CRLB by a large factor. The point at which the threshold occurs as well as its magnitude are factors of obvious practical interest since system performance predictions 
generally are based on the small error assumption (i.e., CRLB) and may, therefore, be extrapolated incorrectly below the threshold yielding extremely optimistic performance predictions.

In the second part of the study we broaden the range of investigation to wideband signals which, for the delay estimation problem, will be divided into two categories: baseband signals and bandpass signals.

In the baseband case, a typical cross correlation response is illustrated in Figure 2 where $W$ denotes signal bandwidth and $[-D / 2, D / 2]$ denotes the a-priori search domain of the unknown delay parameter. One immediately observes a similar, though not as critical, ambiguity phenomenon whenever the a-priori search domain contains at least several peaks of the

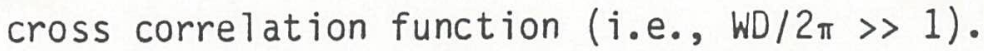

In the bandpass case a typical cross correlation response is illustrated in Figure 3. Here we observe two types of ambiguities: the more critical ambiguity problem results from the highly oscillatory nature of the phase of the observed cross correlation function. A secondary ambiguity phenomenon results from the oscillatory nature of the envelope of the cross correlation function.

The purpose of this paper is to analyze the threshold effects and the attainable m.s.e. for these two classes of signals.

B. Problem Formulation and Assumptions

The basic system of interest here consists of a stationary source radiating a noise-like signal towards two spatially separated receivers. Each receiver also receives an additive noise component so that the actual waveforms observed at the receiver outputs are given by 


$$
\begin{aligned}
& r_{1}(t)=s(t)+n_{1}(t) \\
& r_{2}(t)=s(t-\Delta \tau)+n_{2}(t)
\end{aligned}
$$

We shall assume that $s(t), n_{1}(t)$ and $n_{2}(t)$ are sample functions from uncorrelated zero-mean Gaussian random processes with spectral densities $S(\omega)$, $N_{1}(\omega)$ and $N_{2}(\omega)$, respectively. Since we are primarily interested in the ambiguity problem in time delay estimation, implying the use of widely separated receivers, the assumption of noise incoherence from receiver to receiver is likely to be satisfied.

We shall further assume that $\Delta \tau$, the receiver-to-receiver delay, is uniformly distributed in the interval

$$
-D / 2 \leq \Delta \tau \leq D / 2
$$

This a-priori domain may come about, perhaps from the known receiver separation and the known velocity of propagation in the medium.

Finally, we shall assume that the observation time $T$ is large compared with the correlation time (inverse bandwidth) of signal and noise, i.e. $W T / 2 \pi \gg 1$. This condition is very generally satisfied in problems of practical interest here.

The problem may now be stated as follows: given the data at the receiver outputs ( $\left.i . e ., r_{i}(t) i=1,2\right)$, characterize the minimum m.s.e. estimate of the delay parameter. Our approach is to set a lower bound on the attainable m.s.e. using the modified ZZLB. This approach is completely independent from the actual estimation method. However, in [10] it is shown that for sufficiently large WT products the cross correlator performance comes 
close to the lower bound below as well as above the threshold. These results establish the modified ZZLB as an extremely tight lower bound for problems of this type while demonstrating that the cross correlator is a nearly optimal instrumentation in both the small and large error regimes.

C. The Modified Ziv-Zakai Lower Bound

The modified ZZLB on the m.s.e. of any estimate $\hat{\Delta \tau}$ of $\Delta \tau$ is given by $([8],[9])$

$$
\overline{\varepsilon^{2}} \geq \frac{1}{D} \int_{0}^{D} x G[(D-x) \operatorname{Pe}(x)] d x
$$

where $G[]$ is a non-increasing function of $x$ obtained by filling the valleys in the function $(D-x) P e(x)$ (see Figure $B-1$ in Appendix $B$ ). $P e(x)$ is the minimum probability of error (achievable by the likelihood ratio test) for deciding whether the true value of the parameter is $\Delta \tau_{0}$ or $\Delta \tau_{1}$ where $\Delta \tau_{1}$ - $\Delta \tau_{0}=x$. In general, a closed analytical form for $P e(x)$ cannot be found. However, in ([9], Appendix A) it has been shown that for $W T / 2 \pi \gg 1, P e(x)$ is very closely approximated by

$$
P e(x) \approx e^{a(x)+b(x)} \Phi(\sqrt{2 b(x)})
$$

where

$$
\begin{aligned}
& a(x)=-\frac{T}{2 \pi} \int_{0}^{\infty} \ln \left(1+\operatorname{SNR}(\omega) \sin ^{2} \omega x / 2\right) d \omega \\
& b(x)=\frac{T}{2 \pi} \int_{0}^{\infty} \frac{\operatorname{SNR}(\omega) \sin ^{2} \omega x / 2}{1+\operatorname{SNR}(\omega) \sin ^{2} \omega x / 2} d \omega
\end{aligned}
$$


and

$$
\operatorname{SNR}(\omega)=\frac{\left[S(\omega) / N_{1}(\omega)\right]\left[S(\omega) / N_{2}(\omega)\right]}{I+S(\omega) / N_{1}(\omega)+S(\omega) / N_{2}(\omega)}
$$

$$
\Phi(y)=\frac{1}{2 \pi} \int_{y}^{\infty} e^{-\mu^{2} / 2} d \mu
$$

We have also included Appendix A to demonstrate that the expression on the right hand side of Eq. (4) is, in fact, a lower bound on $\mathrm{Pe}(\mathrm{x})$. Hence, by substituting Eq. (4) into Eq. (3) the inequality sign is preserved.

It is important to observe that since Eq. (7) uses arbitrary spectral functions, the lower bound can be applied to investigate a wide class of signals. As pointed out before, the study will be separated into two parts: in Section II we consider baseband signals. Analytically it appears to be a simpler case and should therefore be understood first. In Section III we consider bandpass signals. In that context all the results concerning narrowband signals [9] will be included and referred to as a special case.

\section{BASEBAND SYSTEMS}

In this section we shall concentrate on signals whose power is distributed about zero frequency. To simplify the form of results, let us consider the following special case

$$
S(\omega)= \begin{cases}S & |\omega| \leq W / 2 \\ 0 & |\omega|>W / 2\end{cases}
$$


If we further assume that the additive noise components are spectrally flat over $[-W / 2, W / 2]$, then Eq. (7) assumes the simplified form

$$
\operatorname{SNR}(\omega)= \begin{cases}\text { SNR } & |\omega| \leq w / 2 \\ 0 & |\omega|>w / 2\end{cases}
$$

where

$$
S N R=\frac{\left(S / N_{1}\right)\left(S / N_{2}\right)}{1+S / N_{1}+S / N_{2}}
$$

and $S / N_{j}$ is the in-band signal-to-noise ratio at the $i^{\text {th }}$ receiver output. Substitution of Eq. (10) into Eqs. (5) and (6) immediately yields

$$
\begin{aligned}
& a(x)=-\frac{T}{2 \pi} \int_{0}^{W / 2} \ln \left(1+\operatorname{SNR} \sin ^{2} \omega x / 2\right) d \omega \\
& b(x)=\frac{T}{2 \pi} \int_{0}^{W / 2} \frac{\text { SNR } \sin ^{2} \omega x / 2}{1+\operatorname{SNR}^{2} \sin ^{2} \omega x / 2} d \omega
\end{aligned}
$$

To obtain the lower bound one must substitute Eqs. (12) and (13) into Eq. (4) and Eq. (4) into Eq. (3) successively, and carry out the indicated algebraic operations. Since we are primarily interested in the ambiguity phenomenon we shall assume that $W D / 2 \pi \gg 1$, say, by at least a factor of 3 . In that case, following some rather extensive algebra manipulations outl ined in Appendix B, it is shown that the lower bound consists of essentially three disjointed segments as suggested by the following equation: 


$$
\overline{\varepsilon^{2}} \geq\left\{\begin{array}{lr}
D^{2} / 12 & (\text { WT } / 2 \pi) \text { SNR }<a \\
\text { THRESHOLD } & \alpha<(\text { WT } / 2 \pi) \text { SNR }<B \\
24 \pi / W^{3} \text { TSNR } & (W T / 2 \pi) \text { SNR }>B
\end{array}\right.
$$

where in the threshold region the lower bound varies essentially exponentially with $(W T / 2 \pi)$ SNR. This result is illustrated in Figure 4 . The lower limit of the threshold region is given by

$$
\alpha=0.92 \simeq-0.36 \mathrm{db}
$$

The upper limit, $B$, is the solution to the following transcendental equation

$$
(B / 2) \Phi(B / 2)=(6 / W D)^{2}
$$

Note that Eq. (16) has two solutions. We only consider the larger one. In Figure 5, $B$ is plotted and tabulated as a function of $W D / 2 \pi$ for the convenience of the reader. Thus in a logarithmic ( $d b$ ) scale $\beta$ varies from about $13 \mathrm{db}$ for moderate WD products to about $16 \mathrm{db}$ for exceedingly large WD products.

To put these results into perspective, we first observe that a m.s.e. of $D^{2} / 12$ corresponds to a random variable uniformly distributed in $[-D / 2$, D/2]. Such a performance level can always be achieved, regardless of signal observations. The first 1 ine in Eq. (14), therefore, reads that for combination of $W T / 2 \pi$ and SNR such that their product (the so-called post-integration SNR) does not exceed $\sim 0 \mathrm{db}$, signal observations are completely dominated by noise thus essentially useless for the delay estimation.

We next observe that m.s.e. predictions based on the Cramer-Rao inequality yield the following lower bound ([9], Appendix B). 


$$
\overline{\varepsilon^{2}} \geq 1 / \frac{T}{\pi} \int_{0}^{\infty} \omega^{2} \operatorname{SNR}(\omega) d \omega
$$

Substituting Eq. (10) into Eq. (17) and carrying out the indicated integration one immediately obtains the third Iine in Eq. (14). Hence, if the post-integration SNR exceeds $B$, the ambiguities in the differential delay observations can essentially be resolved. Cross correlating the received signals one obtains a differential delay estimate whose m.s.e. is closely characterized by the CRLB.

In the derivation of Eq. (14) we have assumed that $W D / 2 \pi \gg>1$. This condition means that the correlation time (inverse bandwidth) of the signal is small compared with the maximum expected receiver-to-receiver delay, or that the spacing between receivers is large compared with half-wavelength of the modulation frequency (signal bandwidth). Only in that case we are dealing with the possibility of a significant ambiguity problem. There remains the question: How the results stated by Eq. (14) are affected when this condition is not satisfied? In Figure 6 the lower bound is generated numerically (by exact integration of Eq. (3)) and plotted as a function of the post-integration SNR (in a logarithmic (db) scale) for fixed WT/2 $\pi$ and different values of $W D / 2 \pi$. The threshold points $\alpha$ and $B$ were calculated using Eqs. (15) and (16). Note that $\overline{\varepsilon^{2}}$ is normalized by $D^{2} / 12$ so that we actually measure the relative efficiency of the attainable m.s.e. to the a-priori variance. One observes a significant threshold effect whenever $W D / 2 \pi$ $>1$. In case $W D / 2 \pi<1$ (i.e. in ambiguity-free situation), one observes a 
rather smooth transition from the CRLB to the $D^{2} / 12$ asymptote. Note, in passing, the close agreement between Eqs. (15) and (16) and the exact limits of the threshold region.

We finally note that in the derivation of Eq. (14) it is assumed that the source signal and the additive noises are spectrally flat over the receiver frequency band. Spectrally flat signal, however, has a highly oscillatory correlation function indicating a serious ambiguity problem. For signal spectra whose correlation function (inverse Fourier transform) is smoothly varying, the ambiguity phenomenon may not be as critical. The lower bound in that case is still composed of the $D^{2} / 12$ segment and the CRLB, but the threshold effect is expected to occur at a lower SNR. This effect can be studied by analyzing the lower bound for various signal and noise spectra.

\section{II . BANDPASS SYSTEMS}

In this section we concentrate on signals whose power is distributed about some center frequency $\omega_{0}$. To simplify the form of results let us consider, in complete analogy with the baseband case, the following special case

$$
S(\omega)= \begin{cases}S & \left|\omega \pm \omega_{0}\right| \leq w / 2 \\ 0 & \left|\omega \pm \omega_{0}\right|>W / 2\end{cases}
$$

We sha11 further assume that the additive noise components are spectrally flat over the signal frequency band so that Eq. (7) assumes the form 


$$
\operatorname{SNR}(\omega)= \begin{cases}\operatorname{SNR} & \left|\omega \pm \omega_{0}\right| \leq W / 2 \\ 0 & \left|\omega \pm \omega_{0}\right|>\cdot W / 2 .\end{cases}
$$

where SNR is defined in Eq. (11). Substitution of Eq. (19) into Eqs. (5) and (6) immediately yields

$$
\begin{aligned}
& a(x)=-\frac{T}{2 \pi} \int_{\omega_{0}-W / 2}^{\omega_{0}^{+W / 2}} \ln \left(1+\operatorname{SNR} \sin ^{2} \omega x / 2\right) d \omega \\
& b(x)=\frac{T}{2 \pi} \int_{\omega_{0}-W / 2}^{\omega_{0}+W / 2} \frac{\text { SNRsin }{ }^{2} \omega x / 2}{1+S_{R} \sin ^{2} \omega x / 2} d \omega
\end{aligned}
$$

The lower bound is now obtained by successive substitutions of Eqs. (20) and (21) into Eq. (4) and Eq. (4) into Eq. (3) and carrying out the indicated algebra operations.

Let us first assume that $W / \omega_{0} \ll 1$. Since we are concerned with the joint effect of envelope and phase ambiguities on the attainable m.s.e. (see Figure 3 ), we shall further assume that $W D / 2 \pi>1$. In this setting, therefore, we are dealing with narrowband signals and very widely separated receivers. Following some rather extensive algebraic manipulations outlined in Appencix C, it is shown that the lower bound consists of essentially two, distinct threshold effects dividing the entire SNR domain into three disjointed segments as suggested by the following equation 


$$
\overline{\varepsilon^{2}} \geq \begin{cases}D^{2} / 12 & (W T / 2 \pi) \text { SNR }<\gamma \\ \text { THRESHOLD } & \gamma<(W T / 2 \pi) \text { SNR }<\delta \\ 12 \pi / W^{3} \text { TSNR } & \delta<(W T / 2 \pi) \text { SNR }<\mu \\ \text { THRESHOLD } & \mu<(W T / 2 \pi) \text { SNR }<\pi \\ \pi / \omega_{0}^{2} W T S N R & (W T / 2 \pi) \text { SNR }>\eta\end{cases}
$$

where in the threshold regions the lower bound varies essentially

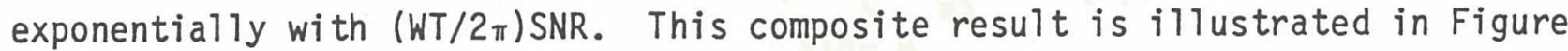
7. The various threshold points will be defined shortly. We further observe that in the narrowband case the CRLB (obtained by substituting Eq. (19) into Eq. (17) and carrying out the indicated integration) is given, to an excellent approximation, by

$$
\overline{\varepsilon^{2}} \geq \pi / \omega_{0} \text { WTSNR }
$$

Thus if $(W T / 2 \pi) S N R>n$, the lower bound coincides with the CRLB. This is the ambiguity free mode of operation.

If $\delta<(W T / 2 \pi) S N R<\mu$, the lower bound exceeds the CRLB by a factor of $12\left(\omega_{0} / W\right)^{2}$. In this region signal observations are subject to unresolved phase ambiguities, however, usefur estimate of the differential delay can still be obtained from the envelope of the cross correlation function. Note that this segment of the lower bound coincides with m.s.e. predictions based on a simplified version of the Barankin lower bound [11]. We further note that the 3rd line in Eq. (14) exhibits exactly $3 \mathrm{db}$ (a factor of 2) loss in m.s.e. relative to the 3 rd line in Eq. (22). This result indicates the following: the 3rd line in Eq. (14) is the CRLB based on the received signal 
envelopes. An estimator which realizes that m.s.e. consists of shifting the received signals to baseband (perhaps because of unresolved phase ambiguities) prior to the cross correlation operation. The latter scheme, therefore, is always inferior to a scheme which first cross correlates the received signals and then uses the envelope of the cross correlation function for the delay estimation.

Finally, if $(W T / 2 \pi)$ SNR $<\gamma$, the lower bound is essentially characterized by the constant level of $D^{2} / 12$. This is the noise dominated region where signal observations are subject to envelope ambiguities as well, thus essentially useless for the delay estimation.

The lower and upper limits of the more critical threshold are given, respectively, by

$$
\begin{aligned}
& \mu=\left(2.76 / \pi^{2}\right)\left(\omega_{0} / W\right)^{2} \\
& \eta=\left(6 / \pi^{2}\right)\left(\omega_{0} / W\right)^{2}\left[\Phi^{-1}\left(W^{2} / 24 \omega_{0}^{2}\right)\right]^{2}
\end{aligned}
$$

where $\Phi^{-1}()$ denotes the inverse of $\Phi()$. Eqs. (24) and (25) depends only on $\omega_{0} / W$, the ratio of center frequency to signal bandwidth. Eq. (25) is of particular interest since it represents the minimum amount of post-integration SNR required to achieve the CRLB. Thus, for example, if $\omega_{0} / W=10$ (i.e. 10 percent signal bandwidth), $\mu=14.5 \mathrm{db}$ and $\eta=28.3 \mathrm{db}$. If $\omega_{0} / \mathrm{W}=100$ (i.e. 1 percent signal bandwidth), $\mu=34.5 \mathrm{db}$ and $n=50.8 \mathrm{db}$. One further observes that the threshold region is not infinitely small as may be interpreted from the analysis based on the Barank in lower bound [11]. For 10 percent signal bandwidth it is a segment of $13.8 \mathrm{db}$. For 1 percent signal 
bandwidth it is a segment of $16.3 \mathrm{db}$.

The lower and upper limits of the secondary threshold are given, respectively, by

$$
\begin{aligned}
& \gamma=\alpha / 2=0.46 \simeq-3.36 \mathrm{db} \\
& \delta=B / 2
\end{aligned}
$$

where $B$ is defined by Eq. (16). Hence, the secondary threshold is shifted by exactly $3 \mathrm{db}$ (a factor of 2 ) relative to the threshold phenomenon found in the baseband case.

The analytical result illustrated in Figure 7 is derived under the assumption that $w / \omega_{0} \ll 1$. In that case, the lower bound exhibits two distinct threshold effects. The phase ambiguities and envelope ambiguities occur at essentially disjointed segments of the SNR domain and therefore, in a sense, strictly additive. This conclusion, however, applies only to narrowband signals. As the signal bandwidth increases the two threshold regions come close (i.e., $\delta$ converges to $\mu$ ) and the two ambiguity phenomena are, in fact, indistinguishable. This effect is illustrated in Figures 8-10 where the lower bound is generated by exact numerical integration of Eq. (3). Note, in passing, the close agreement between the various threshold points, calculated using Eqs. (24) to (27), and the exact 1 imits of the threshold regions. 


\section{Narrowband Systems}

It is not difficult to relate the composite result given by Eq. (22) to the analys is of narrowband systems carried out in [9]. In [9] the lower bound is derived under the assumption that the signal bandwidth is so narrow that its correlation time (inverse bandwidth) exceeds the maximum expected delay (i.e. $W D / 2 \pi<1)$. The envelope-type ambiguities, therefore, are completely el iminated from considerations. Instead of having a threshold effect, there is a smooth transition from the first line of $\mathrm{Eq}$. (22) to its third line as illustrated in Figure 11. The point at which the transition occurs (denoted by $\rho$ in the figure) is obtained by simply intersecting the two indicated lines. One finds

$$
\rho=\left(18 / \pi^{2}\right) /(W D / 2 \pi)^{2}
$$

With this modification Eq. (22) reduces to the result derived in [9].

\section{CONCLUSIONS}

This is the second part of a study which deals with the problem of passive time delay estimation. The focus here is on systems employing wideband signals and/or arrays of very widely separated receivers. A modified (improved) version of the ZZLB is used to analyze the effect of additive noise and signal ambiguities on the attainable mean square estimation errors.

We first concentrated on baseband (lowband) signals. When the lower bound is plotted as a function of SNR, one observes a distinct threshold phenomenon. Above a critical SNR the lower bound approaches the CRLB. This 
is the small error region. Below the threshold, the lower bound quickly approaches a constant level depending only on the variance of the a-priori search domain of the delay parameter. In this region signal observations are completely dominated by noise, thus essentially useless for the delay estimation. Information concerning the threshold points and the various segments of the lower bound is given by Eq. (14).

Delay estimation using bandpass signals is analytically a more complicated problem. Here the lower bound exhibits two distinct threshold effects dividing the SNR domain into three disjointed segments: at high SNR the lower bound coincides with the CRLB. This is the ambiguity-free mode of operation where differential delay estimation is subject only to local

errors. At moderate SNR (between the two thresholds) the lower bound exceeds the CRLB by a factor of $12\left(\omega_{0} / W\right)^{2}$ where $\omega_{0}$ and $W$ are, respectively, the center frequency and signal bandwidth. In this region the ambiguities in the received signal phases cannot be resolved, however, a useful estimate of the differential delay can still be obtained from the received signal envelopes. At low SNR the lower bound approaches a constant level depending only on the variance of the a-priori search domain of the delay parameter. In this region, signal observations are subject to envelope ambiguities as well, thus essentially useless for the delay estimation. Information concerning the various threshold points and the various segments of the lower bound is given by Eq. (22). 
Acknowledgements

The authors would like to thank Dr. J.P. Ianniello and Professor P.M. Schultheiss for their helpful comments and discussions throughout the course of study, and to Mrs. Ann Henry for her patient and excellent secretarial assistance. This work was performed under the Naval Underwater Systems Center Contract No. N00140-83-C-KA35. 
$-18-$

APPENDIX A Lower Bounds on $P_{e}(x)$

The binary decision problem under consideration here is given by

$$
\begin{aligned}
& H_{0}: \Delta \tau=\Delta \tau_{0}=a \\
& H_{1}: \angle \tau=\angle \tau_{1}=a+x
\end{aligned}
$$

The $\log$ likelihood ratio test ( $\log$ LRT) between $H_{0}$ and $H_{1}$ is defined by

$$
l=\log \frac{R\left(\Sigma \mid H_{1}\right)}{R\left(\underline{r} \mid H_{0}\right)}
$$

where $f\left(r \mid H_{i}\right)$ is the conditional probability density of $\underline{r}$ under $H_{i}$ hypothesis, and $r$ represents the data vector (e.g. time samples or Fourier coefficients of the received signals). Assuming that $H_{0}$ and $H$, are equally likely to occur (ie.

$\left.P\left(H_{0}\right)=P\left(H_{1}\right)=/ 2\right) \quad$ a decision rule which minimizes the probability of error compares the log LRT to zero threshold.

If $\ell \geqslant 0$ we decide on $H_{1}$, if $l<0$ we decide on $H_{0}$.

Hence, the minimum attainable probability of error is given by

$$
P_{e}(a, a+x)=\frac{1}{2} \int_{0}^{\infty} R\left(l ; H_{0}\right) d l+\frac{1}{2} \int_{-\infty}^{0} R\left(l \mid H_{1}\right) d l
$$

Let $\Psi_{i}(S)$ denotes the characteristic function associated with $R\left(f \mid H_{i}\right) \cdot R\left(\ell \mid H_{i}\right)$ and $Y_{i}(s)$ are, by definition, a Fourier transform pair satisfying 
$p\left(l \mid H_{0}\right)=\frac{1}{2 \sqrt{j} j} \int^{c_{0}+y_{\infty}} y_{0}(s) e^{-s l} d s$.

$$
c_{0} \cdot j \infty
$$

and

$$
\left.R^{\prime} l \mid H_{1}\right)=\frac{1}{2 \pi j} \int_{c_{1}-j \infty}^{c_{1}+j \infty} Y_{1}(s) e^{-s l} d s
$$

The integrals in Eqs. $(\mathrm{A}-4)$ and (A-5) must be evaluated at $C_{0}=0$ and $C_{1}=0$, respectively. However, for future manipulations, let $C_{0}$ be a real-valued parameter in the open interval $(0,1)$. Similarly, let $C_{1}$ be a real-valued parameter in $(-1,0)$. In that case, substituting Eqs. (A-4) and (A-5) into Eq. (A-3), one obtains

$$
\begin{aligned}
& P_{l}(a, a-x)=\frac{1}{2} \cdot \frac{1}{2 \pi j} \int_{c_{0}-j^{\infty}}^{c_{0}+i \infty} \psi_{0}^{i}(s) d s \int_{0}^{\infty} e^{-s l} d l+\frac{1}{2} \cdot \frac{1}{2 \pi j} \int_{c_{1}-j^{\infty}}^{c_{1}+j \infty} y_{1}(s) d s \int_{-\infty}^{n} e^{-s l} d l \\
& =\frac{1}{2} \cdot \frac{1}{2 \pi j} \int_{c_{0}-j \infty}^{c_{0}+j \infty}\left[\Psi_{0}(s) / s\right] d s-\frac{1}{2} \cdot \frac{1}{2 \pi_{1}} \int_{c_{1}-j_{0} \infty}^{c_{1}+j \infty}\left[\psi_{1}(s) / s\right] d s
\end{aligned}
$$

Note, in passing, that the transition from the first version of Eq. (A-6) to its second version can be carried out if and only if $c_{0}>0$ and $c_{1}<0$. In this setting $P_{\ell}(a, a+x)$ is expressed in terms of $Y_{i}(S), i=0,1$. Now, by definition

$$
\Psi_{i}(s) \triangleq E\left\{e^{s l} \mid H_{i}\right\} \quad i=0,1
$$

where $E\{\}$ denotes the statistical expectation of the bracketed quantity. Substituting Eq. (A-2) into Eq. (A-7) and observing that $l=\ell(r) \quad$ (so that the statistical expectation operation can be 
performed with respect to the probability density of $\boldsymbol{r}$ ), one immediately obtains

$$
\begin{aligned}
& \psi_{0}(s)=\int_{-\infty}^{\infty}\left[R\left(\underline{r} \mid H_{1}\right)\right]^{s}\left[R\left(\underline{r} \mid H_{0}\right)\right]^{1-s} d \underline{r} \\
& \psi_{1}(s)=\int_{-\infty}^{\infty}\left[R\left(\underline{r} \mid H_{1}\right)\right]^{1+s}\left[R\left(\underline{r} \mid H_{0}\right)\right]^{-s} d \underline{r}
\end{aligned}
$$

We shall now generate the data vector $\underline{r}$ by Fourier analyzing $r_{k}(t)$ in Eq. (1). Since signal and noise are assumed to be zero mean Gaussian processes and the components of $r$ are generated by linear operations on these time functions, $\underline{v}$ has a multivariate Gaussian distribution

$$
p\left(r \mid H_{i}\right)=\frac{1}{\operatorname{dut}\left(\pi k_{i}\right)} \cdot \exp \left(-\underline{r}^{* *} k_{i}^{-1} \underline{r}\right) \quad j i=0,1
$$

where

$$
K_{i}=E\left\{r^{*} \mid H_{i}\right\} \quad i=0,1
$$

and $r^{*}$ denotes the conjugate transpose of $\underline{r}$. To obtain $\Psi_{0}(s)$ one must substitute Eq. (A-10) into Eq. (A-8) and carry out the indicated algebra operations. For observation time $T$ large compared with the correlation time (inverse bandwidth) of signal and noise (i.e. $W T / 2 \pi>>1$ ), 
the Fourier coefficients (at each receiver output) associated with different frequences are statistically uncorrelated. In that case the required computations become relatively easy. Details are contained in ( [9], Appendix A). The result is

$$
y_{0}(s)=\prod_{k}\left[1+4 s(1-s) \Gamma\left(\omega_{k}, x\right)\right]^{-1}
$$

where

$$
U_{k}=2 \pi K / T
$$

and

$$
\Gamma\left(\omega_{k}, x\right)_{-}=\operatorname{SNR}\left(\omega_{k}\right) \sin ^{2} \omega_{k} x / 2
$$

The index $k$ varies over all components in the signal frequency band. Following very similar considerations for $Y_{1}(S)$ one obtains

$$
Y_{1}(s)=\prod_{k}\left[1-4 s(1+s) \Gamma\left(\omega \cup_{k}, x\right)\right]^{-1}
$$

Note that $\Psi_{0}(S)$ and $\Psi_{1}(S)$ depend only on $x$ so that $P_{e}(a, a+x)=P_{e}(x) \quad$. One must now substitute Eqs. $(A-12)$ and $(\mathrm{A}-15)$ into Eq. $(\mathrm{A}-6)$ and carry out the indicated integration. It can be easily shown that all the poles (singular points) of $\psi_{0}(S)$ are located on the real axis outside the interval $[0,1]$, all the poles of $\Psi_{,}(S)$ are located on the real axis outside the interval $[-1,0]$. Hence, the first integral in Eq. (A-6) can be evaluated for any value of $C_{0}$ in the open interval $(0,1)$ without affecting the desired result. Similarly, the 
- $22-$

second integral in Eq. $(A-6)$ can be evaluated for any value of $C_{\mathbf{1}}$ in $(-1,0)$. We shall find it most convenient to choose $C_{0}=1 / 2, C_{1}=-1 / 2$. We shall further make the change of variables $S=\frac{1}{2}+y^{\prime} y$ in the first integral and $s=-\frac{1}{2}+j y$ in the second integral. In this setting one obtains

$$
\begin{aligned}
& D_{e}(x)=\frac{1}{2} \frac{1}{2 \pi} \int_{-\infty}^{\infty} \frac{1}{1 / 2+j y}\left\{\prod_{x}\left[1+4\left(\frac{1}{2}+j y\right)\left(\frac{1}{2}-j y\right) i\left(\omega_{k}, x\right) j^{-1}\right\} d y\right. \\
& -\frac{1}{2} \frac{1}{2 \pi} \int_{-\infty}^{\infty} \frac{1}{-r_{2}+y y}\left\{\prod_{k}\left[1+4\left(\frac{1}{2}+j y\right)\left(\frac{1}{2}-y y\right) \Gamma\left(\omega_{k}, x\right)\right]^{-1}\right\} d y \\
& =\frac{1}{\pi} \int_{-\infty}^{\infty}\left\{\pi\left[1+1+4 y^{2}\right) \Gamma\left(\omega_{k}, x\right)^{-1}\right\} \frac{d y}{1+4 y^{2}} \\
& =\frac{2}{\pi}\left\{\prod_{k}\left[1+\Gamma\left(\omega_{k}, x\right)^{-1}\right\} \int_{-\pi}^{\infty} \int_{0}^{\infty}\left[1+\frac{\Gamma\left(\omega_{k}, x\right)}{1-\Gamma\left(\omega_{k}, x\right)} 4 y^{2}\right\}^{-1} \frac{c^{c} y}{1+4 y^{2}}(A-16)\right. \\
& \text { This is an exact expression for } P_{\ell}(x) \text {. To generate a lower } \\
& \text { bound on } P_{e}(x) \text { we shall use the inequality }(1+x)^{-1} \geqslant e^{-x} \text { whenever } \\
& x \geqslant 0 \text {. Thus } \\
& {\left[1+\frac{\Gamma\left(\omega_{k}, x\right)}{1+\Gamma\left(\omega_{k}, x\right)} H y^{2}\right]^{-1} \geqslant \exp \left\{-\frac{\Gamma\left(\omega_{k}, x\right)}{1+\Gamma\left(\omega_{k}, x\right)} 4 y^{2}\right\}} \\
& \text { Substituting Eq.(A-17) into Eq. (A-16) one obtains } \\
& P_{e}(x) \geqslant\left\{\prod_{k}\left[1+\Gamma\left(\omega_{k}, x\right)\right]^{-1}\right\} \frac{2}{\pi} \int_{0}^{\infty} \exp \left\{-4 y^{2} \sum_{k} \frac{\Gamma\left(\omega_{k, x}\right)}{1+\Gamma\left(\omega_{k}, x\right)}\right\} \frac{d y}{1+4 y^{2}} \\
& =e^{a(x)} \frac{2}{\pi} \int_{0}^{\infty} e^{-4 y^{2} b(x)} \frac{d y}{1+4 y^{2}}
\end{aligned}
$$


where we defined

$a(x)=-\sum_{k} \ln \left[1+\Gamma\left(\omega_{k}, x\right)\right]$

$b(x)=\sum_{k} \frac{\Gamma\left(\omega_{k}, x\right)}{1+\Gamma\left(\omega_{k}, x\right)}$

The integral in Eq. (A-18) can be evaluated analytically using [12], p. 338, formula 3,466-1. The result is given by

$$
=(x) \geqslant e^{a(x)+b(x)} \phi(\sqrt{2 b(x)})
$$

which is the desired result. Substituting Eq. (A-14) into Eqs. (A-19) and $(A-20)$, one obtains

$$
\begin{aligned}
& \left.c^{\prime}(x)=-\sum_{k} \ln \left[1+\operatorname{sik} \omega_{k}\right) \cdot \sin ^{2} \omega_{k} x / 2\right] \\
& =-\frac{T}{2 \pi} \sum_{k} \ell_{n}+\operatorname{SNR}_{n}\left(\omega_{n}\right) \cdot \operatorname{sen}^{2} \omega_{k} x / 2 \Delta \cdot \Delta \\
& \left.\delta^{\prime} x\right)=\sum_{k_{1}} \frac{\operatorname{sNR}\left(\omega_{k}\right) \cdot \sin ^{2} \omega_{k} x / 2}{1+\operatorname{SNR}\left(\omega_{k}\right) \cdot \sin ^{2} \omega_{k} x / 2} \\
& =\frac{T}{2 \pi} \sum_{k} \frac{\operatorname{SNR}\left(\omega_{n}\right) \cdot \sin ^{2} \omega_{k x} / 2}{1+\operatorname{SNR}\left(\omega_{k}\right) \cdot \sin ^{2} \omega_{k} x / 2} \cdot \Delta \omega
\end{aligned}
$$

where we define $\Delta \omega=2 \pi / T$. For large WT froduct and smoothly varying signal and noise spectra the function $S N R(\omega) \sin ^{2} \omega x_{2}$ changes only insignificantly over the frequency increment of $\Delta \omega$, so that the sums in Eqs. (A-22) and (A-23) can be converted into the integrals in Eqs. (5) and (6), respectively.

Eq. (A-21) can further be used to generate weaker lower bounds 
$-24-$

on $\mathrm{Pe}(\mathrm{x})$, which will become useful in the proceeding analysis. We shall start from Eqs. $(A-19)$ and $(A-20)$. Since

$$
\begin{aligned}
& \frac{\Gamma\left(\omega_{k}, x\right)}{1+\Gamma\left(\omega_{k}, x\right)} \leqslant \Gamma\left(\omega_{k}, x\right) \\
& \text { and since } \\
& \ln \left[1+\Gamma\left(\omega_{k}, x\right)\right]-\frac{\Gamma\left(\omega_{k}, x\right)}{1+\Gamma\left(\omega_{k}, x\right)} \leqslant \frac{1}{2} \Gamma^{2}\left(\omega_{k}, x\right) \\
& \quad \text { It immediately follows that } \\
& 2 b(x) \leqslant 2 \sum_{k} \Gamma\left(\omega_{k}, x\right)
\end{aligned}
$$

and

$$
a(x)+b(x) \geqslant-\frac{1}{2} \Gamma^{2}\left(u_{k}, x\right)
$$

$(A-27)$

where $2 b(x)$ and $[a(x)+b(x)]$ are the terms appearing in Eq. $(A-21)$. Replacing the first term by its upper bound, and the second term by its lower bound, $P_{e}(x)$ can further be bounded by

$$
P_{e}(x) \geqslant e^{-d(x)} \phi(\sqrt{c(x)})
$$

where $c(x)$ and $d(x)$ are given by 
$-25-$

$$
\begin{aligned}
& C(x)=2 \sum_{k} \Gamma\left(\omega_{k}, x\right)=\frac{T}{\pi} \sum_{k} \sin R\left(\omega_{k}\right) \sin ^{2}\left(\omega_{k} x / 2\right) \Delta \omega \\
& \underset{\omega T / 2 R \partial 1}{\longrightarrow} \frac{T}{\pi} \int_{0}^{\infty} \operatorname{SNR}(\omega) \cdot \sin ^{2}(\omega x / 2) d \omega \\
& d(x)=\frac{1}{2} \sum_{k} \Gamma^{2}\left(\omega_{k}, x\right)=\frac{T}{4 \pi} \sum_{k}\left[\operatorname{SNR}\left(\omega_{k}\right) \cdot \sin ^{2}\left(\omega_{k} x / 2\right)\right]^{2} \Delta \omega \\
& \underset{\omega T / 2 \pi \gg 1}{\longrightarrow} \frac{T}{4 \pi} \int_{0}^{\infty}\left[\operatorname{SNR}(\omega) \cdot \sin ^{2} \omega x / 2\right]^{2} d \omega \\
& (A-30) \\
& c(x) \leqslant c^{2} x^{2} \\
& d(x) \leqslant d^{4} x^{4}
\end{aligned}
$$

where

$$
c^{2}=\frac{T}{4 \pi} \int_{0}^{\infty} \omega^{2} \operatorname{SNR}(\omega) d \omega
$$

and

$$
d^{\nu}=\frac{T}{64 \pi} \int_{0}^{\infty}\left[\omega^{2} \operatorname{SNR}(\omega)\right]^{2} d \omega
$$

Substituting $c(x)$ and $d(x)$ by their upper bounds, one immediately obtains

$$
P_{e}(x) \geqslant \varepsilon^{-d^{4} x^{4}} \phi(c x)
$$


We note, in passing, that the lower bound given by Eq. (A-35) is useful only for small $x$, where the inequalities in Eqs. $(A-31)$ and $(\mathrm{A}-32)$ are tight. 
Appendix B Analysis of the Modified ZZLB For Baseband Signals

The modified ZZLB is given by Eq. (3), rewritten here for

reference

$\overline{\epsilon^{2}} \geqslant \frac{1}{D} \int_{0}^{D} x G\left[(D-x) P_{e}(x)\right] d x$

The function $G\left[(D-x) P_{e}(x)\right]$ assumes a simple form if

$P_{e}(x)$ contains several well-defined peaks. This is illustrated in Figure

12 for a typical baseband case. Thus, if $x_{0}, x_{1}, x_{2}, \ldots$ are the local

maxima of $P_{l}(x)$, then at each segment $\left[x_{n-1}, x_{n}\right]$ the function

$G\left[(D-x) P_{e}(x)\right]$ is closely bounded by

$G\left[(D-x) P_{e}(x)\right] \geqslant\left(D-x_{n}\right) P_{e}\left(x_{n}\right) \quad x_{n-1} \leqslant x \leqslant x_{n}$

Note that since $G\left[(D-x) P_{e}(x)\right]$ is a non-increasing function of $x$, Eq. (B-2) holds for arbitrary set of $x_{n}^{\prime} s$. We further note that the local maxima of $P_{e}(x)$ are, in fact, the local maxima (ambiguity points) of the cross-correlation function. These occur, approximately, at (see Figure 2).

$$
\begin{aligned}
& x_{n}=(2 \pi / w) n \quad n=0,1,2, \cdots \\
& P_{e}\left(x_{n}\right) \text {, required for the computation, of Eq. } \quad(B-2) \text {, is }
\end{aligned}
$$
closely approximated using the expression in Eq. (4). Following the derivation in Appendix A, that expression is shown to be a lower bound. 
$-28-$

Thus

$$
P_{e}\left(x_{n}\right) \geqslant e^{a\left(x_{n}\right)+b\left(x_{n}\right)} \phi\left(\sqrt{2 b\left(x_{n}\right)}\right)
$$

where $a\left(x_{n}\right)$ and $O\left(x_{n}\right)$ are obtained by substituting $x=x_{n}$ into Eqs.

(12) and (13), respectively. One obtains

$$
\begin{aligned}
a\left(x_{n}\right) & =-\frac{T}{2 \pi} \int_{0}^{W / 2} \ln \left(1+\operatorname{SNR} \cdot \sin ^{2} \omega x_{n} / 2\right) d \omega \\
& =-\frac{w T}{2 \pi} \frac{1}{W x_{n} / 2} \int_{0}^{W x_{n} / 4} \ln \left(1+\operatorname{SNR} \sin ^{2} \Omega\right) d \Omega
\end{aligned}
$$

and

$$
\begin{aligned}
b\left(x_{n}\right) & =\frac{T}{2 \pi} \int_{0}^{w / 2} \frac{\operatorname{SNR} \cdot \sin ^{2} \omega x_{n} / 2}{1+\operatorname{SNR} R \cdot \sin ^{2} \omega x_{n} / 2} d \omega \\
& =\frac{w T}{2 \pi} \frac{1}{w x_{n} / 2} \int_{0}^{w x_{n} / 4} \frac{S N R \cdot \sin ^{2} \Omega}{1+S N R \cdot \sin ^{2} \Omega} d \Omega
\end{aligned}
$$

where $1 / x_{n} / 4=n \pi / 2$. Since the integrands in Eqs. (B-5) and (B-6) are even and periodic functions of $\Omega$ with a period of $\pi$, and since we are integrating over $[0, n \pi / 2]$, these equations become independent of $n$, i.e. $a\left(x_{n}\right)=a$ and $b\left(x_{n}\right)=b$, where

$$
\begin{aligned}
& a=-\frac{w T}{2 \pi} \cdot \frac{1}{\pi} \int_{0}^{\pi / 2} \ln \left(1+\operatorname{siR} \sin ^{2}-2\right) d \Omega=-\frac{W T}{2 \pi} \ln \frac{1+\sqrt{1+\operatorname{SiR} R}}{2}(B-7) \\
& b=\frac{w T}{2 T} \frac{1}{\pi} \int_{0}^{\pi / 2} \frac{S N R \cdot \sin ^{2} \Omega}{1+S N R \cdot \sin ^{2} \Omega} d \Omega=\frac{W T}{4 \pi} \frac{\sqrt{1+S N R}-1}{\sqrt{1+S N R}}
\end{aligned}
$$


The integral appearing in Eq. $(B-7)$ can be found in [12], p. 594 formula 4.399. The integral appearing in Eq. (B-8) can be modified to a form found in [12], p. 152, formula 2.562. Thus

$P_{e}\left(x_{n}\right) \geqslant e^{a+b} \phi(\sqrt{2 b})$

Substituting Eqs. (B-3) and (B-9) into Eq. (B-2), one obtains

$$
\begin{aligned}
&\left.G[D-x) P_{e}(x)\right] \geqslant\left(D-x_{n}\right) 2^{a+b} \phi(\sqrt{2 b}) \quad x_{n}-2 \pi / w \leqslant x \leqslant x_{n} \quad \text { (B-10) } \\
& \text { Since } D-x_{n} \geqslant D-2 \pi / w-x \text { for all } x \in\left[x_{n}-2 \pi / w, x_{n}\right] \text {, it immediately }
\end{aligned}
$$

follows that

$C-\left[(D-x) \partial_{e}(x)\right] \geqslant\left\{\begin{array}{cc}\left.(D-2 \pi / w-x) e^{a+b} \phi^{(} \sqrt{2 b}\right) & C \leqslant x \leqslant D-2 \pi / w(B-11) \\ 0 & D-2 \pi / w \leqslant x \leqslant D\end{array}\right.$

The first line in Eq. $(B-11)$ is illustrated by the dashed line in Figure 12. The second line in Eq. (B-11) indicates that zero is a better bound. For $W D / 2 \pi \gg 1$, the lower bound presented by Eq. (B-11) is very tight except for values of $x$ in the vicinity of $X=0$, where $P_{l}(x)$ changes rapidly. To take this effect into account, we observe that for small $x$, $P_{l}(x)$ is closely bounded using Eq. $(A-35)$. In that region, therefore, $G\left[(D-\lambda) P_{e}(x)\right]$ is closely bounded by 
$G\left[(D-x) P_{e}(x)\right] \geqslant G\left[(D-x) e^{-d^{4} x^{4}} \phi(c x)\right]$

$$
=(D-x) e^{-d^{4} x^{4}} \phi(c x)
$$

where in the transition from the first version of Eq. (B-12) to its second version we observe that $G[f(x)]=f(x)$ whenever $f(x)$ is a non-increasing function of $x$. The parameters $c$ and $d$ are obtained by substituting Eq. (10) into Eqs. $(A-33)$ and $(A-34)$, respectively. One obtains

$$
c^{2}=\frac{T}{4 \pi} \int_{0}^{\omega / 2} \omega^{2} S_{N} R d \omega=\frac{\omega^{3} T S N R}{96 \pi}
$$

and

$$
d^{4}=\frac{T}{64 \pi} \int_{0}^{\omega / 2}\left[\omega^{2} S N R\right]^{2} d \omega=\frac{\omega^{5} T S N R^{2}}{5 \cdot 2^{\prime \prime} \cdot \pi}
$$

For future manipulations we note that

$$
(c / d)^{4}=\frac{20}{9}(W T / 2 \pi)
$$

$$
\text { Combining Eqs. }(B-11) \text { and }(B-12), G\left[(D-x) P_{l}(x)\right] \text { is tight } 1 y
$$

bounded by

$$
G\left[(D-x) P_{e}(x)\right] \geqslant\left\{\begin{array}{ll}
(D-x) e^{-d^{4} x^{4}} \phi(c x) & 0 \leqslant x \leqslant z \\
(D-2 \pi / w-x) e^{a+b} \phi(\sqrt{2 b}) & z<x \leqslant D-2 \pi / w \\
0 & D-2 \pi / w<x \leqslant D
\end{array}\right. \text { (B-16) }
$$

where $z$ is defined by 


$$
c z=\sqrt{2 b}
$$

Note that $g$ is approximately the point at which the first and second lines in Eq. (E-16) intersect. Substituting Eq. (B-16) into Eq. (B-1) one obtains the following lower bound

$\overline{\epsilon^{2}} \geqslant \frac{1}{D} \int_{0}^{\delta} x(D-x) e^{-d^{4} x^{4}} \phi(c x) d x+\left[\frac{1}{D} \int_{z}^{D-2 \pi / w} x(D-2 \pi / w-x) d x\right] e^{a+b} \phi(\sqrt{2 b})$

Carrying out some straight forward algebra manipulations, it can easily be shown that $z<2 \pi / w$. Since we are assuming that $2 \pi / w<D$, the first term on the right hand side of Eq. (B-18) is closely approximated by $\frac{1}{D} \int_{0}^{3} x(D-x) e^{-d^{4} x^{4}} \phi(c x) d x \approx \int_{0}^{3} x e^{-d^{4} x^{4}} \phi(c x) d x$ $=\frac{1}{c^{2}} \int_{0}^{c} x \operatorname{2xp}\left\{-\frac{x^{4}}{(c / d)^{4}}\right\} \phi(x) c l x=\frac{1}{c^{2}} \int_{0}^{\sqrt{2 b}} x \cdot \exp \left\{-\frac{9 x^{4}}{20(w / 2 \pi)}\right\} \phi(x) d x$

where in the transition from the second versions of Eq. (B-19) to its third version we have substituted Eqs. (B-15) and (B-17). Similarly, the integral appearing in the second term of Eq. (B-18) is closely approximated by

$$
\frac{1}{D} \int_{z}^{D-2 \pi / w} x(D-2 \pi / w-x) d x \approx D^{2} / 6
$$

$$
\text { Substituting Eqs. }(B-19) \text { and (B-20) into Eq. (B-18), one }
$$

obtains 
$\overline{E^{2}} \geqslant \sim \frac{1}{c^{2}} \int_{0}^{\sqrt{2 b}} x \cdot \exp \left\{-\frac{9 x^{4}}{20(w T / 2 \pi)}\right\} \phi(x) d x+\frac{D^{2}}{6} e^{a+b} \phi(\sqrt{2 b})$

Numerical integration indicates that Eq. (B-21) is an excellent approximation to the exact result (Eq. (B-1)). The lower bound presented by Eq. ( $(B-21)$ exhibits two distinct asymptotes: As $S N R \rightarrow 0$, the variables $a, b$ and $c$ approaches zero. In that limit the second term in Eq. (B-21) becomes the dominant term and the lower bound approaches

$$
\overline{\epsilon^{2}}>D^{2} / 12
$$

As $\leq N R \rightarrow \infty \quad, a+b \longrightarrow-\infty$ and $b \longrightarrow w T / 4 \pi$. In that limit the first term in Eq. (B-21) becomes the dominant term in the sum and the lower bound approaches

$$
\begin{aligned}
& \overline{\epsilon^{2}} \geqslant \frac{1}{c^{2}} \int_{0}^{\sqrt{w T / 2 \pi}} x \cdot \exp \left\{-\frac{9 x^{4}}{20(w T / 2 \pi)}\right\} \phi(x) d x \stackrel{W T / 2 \pi>>1}{\longrightarrow} \\
& \frac{1}{c^{2}} \int_{0}^{\infty} x \phi(x) d \psi=\frac{1}{4 c^{2}}=\frac{12}{w^{2}} \cdot \frac{1}{(w T / 2 \pi) S N R}
\end{aligned}
$$

One immediately identifies Eq. (B-22) with the first line of

Eq. (14), and Eq. (B-23) with its third line.

The transition from the $D^{2} / 12$ asymptote to the

$1 / 4 c^{2}$ asymptote essentially starts when

$$
e^{a+b} \phi(\sqrt{2 b})=\frac{1}{4}
$$


and is essentially completed when

$\frac{D^{2}}{6} e^{a+b} \phi(\sqrt{2 b})=1 / 4 c^{2}$

where in the transition region (the so-called threshold region) the lower bound varies essentially as $\phi(\sqrt{2 b})$. One must now substitute Eqs. $(B-7),(B-8)$ and $(B-13)$ into Eqs. $(B-24)$ and $(B-25)$, and solve these equations with respect to SNR in order to obtain the corresponding $3 \mathrm{db}$ points. Suppose, for the moment, that these solutions are obtained at SNR $\ll 1$. In that case, Eqs. $(B-7)$ and (B-8) can be approximated, without incurring any significant errors, by

$$
\begin{aligned}
& a \approx-\frac{W T}{2 \pi} \ln (1+S N R / H) \approx-\frac{W T}{2 \pi} \cdot \frac{S N R}{4} \\
& b \approx \frac{W T}{4 \pi} \cdot \frac{S N R}{2}
\end{aligned}
$$

With these approximations, Eqs. $(B-24)$ and $(B-25)$ assume the simplified forms

$$
p(\sqrt{R / 2})=1 / 4
$$

and

$$
R / 2 \phi(\sqrt{R / 2})=(G / W D)^{2}
$$

where $R$ is the post-integration SNR defined by 


$$
R=(\omega T / 2 \pi) S N R
$$

Denoting by $R=\alpha$ the solution to Eq. (B-28) and substituting $2\left[\phi^{-1}(1 / 4)\right]^{2}=0.92$, one immediately obtains Eq. (15). Denoting by $R=\beta$ the solution to Eq. (B-29), one immediately obtains Eq. (16). We further observe that in terms of SNR the solution to Eq. (B-28) reads $S N R=\alpha /(W T / 2 \pi)$, the solution to $E q \cdot(B-29)$ reads $S N R=\beta /(\omega T / 2 \pi)$. Thus, for $W T / 2 \pi \gg 1$, the simplifying approximations made in Eqs. $(B-26)$ and $(B-27)$ may affect these results only insignificantly. 
Appendix C. Analysis Of The Modified ZZLB For Bandpass Signals.

The modified ZZLB is given by Eq. (3), rewritten here for reference.

$$
\bar{E}^{2} \geqslant \frac{1}{D} \int_{0}^{D} x \in\left[(D-x) P_{e}(x)\right] d x
$$

For values of $x$ in the vicinity of $x=0$, the function $\left.C-[D-x) P_{e}(x)\right]$ is closely bounded using Eq. (B-12), where the parameters $c$ and $d$ are obtained by substituting Eq. (19) into Eqs. (A-33) and (A-34), respectively. Assuming that $\omega_{0} / w \gg 1$ (so that one can ignore terms on the order of $\left(w / \omega_{0}\right)^{2}$ relative to 1$), c^{2}$ and $d^{4}$ are given, respectively, by

$$
\begin{aligned}
& c^{2}=\frac{W T}{4 \pi} \omega_{0}^{2} S N R \\
& d^{4}=\frac{W T}{64 \pi} \omega_{0}^{4} S N R^{2}
\end{aligned}
$$

For future manipulations we note that

$$
(c / d)^{4}=8(w T / 2 \pi)
$$

For values of $x$ away from $x=0$, a tighter lower bound on $G\left[(D-x) P_{e}(x)\right] \quad$ can be generated from the local maxima of $P_{e}(x)$. This is illustrated in Figure 12 for a typical baseband case. In the bandpass case, however, $P_{e}(x)$ has two sets of local maxima. One set, $X_{n}=(2 \pi / w)_{n}$ 
$n=1,2, \ldots \quad$, is associated with the ambiguities in the envelope of the cross-correlation function, the other set, $\tilde{x}_{n}=\left(2 \pi / \omega_{0}\right) n$, is associated with the ambiguities in the phase of the cross-correlation function.

We shall assume, without any significant loss in generality that $\omega_{0} / \omega=K$ is an integer. In that case, using the set $x_{n}=(2 \pi / h) n$ and following the same considerations outlined in Appendix $B, G\left[(D-x) P_{e}(x)\right]$ is bounded using Eq. (B-11), where a and b are given by

$$
a=-\frac{w T}{\pi} \ln \frac{1+\sqrt{1+S N R}}{2}
$$

$$
b=\frac{W T}{2 \pi} \frac{\sqrt{1+S N R}-1}{\sqrt{1+S N R}}
$$

Note that there is a factor of 2 difference between Eq. (C-5) and Eq. (B-7), and between Eq. (C-6) and Eq. (B-8). We shall now use the set $\tilde{x}_{n}=\left(2 \pi / \omega_{0}\right) n$ to generate another lower bound on $G\left[(D-x) P_{e}(x)\right.$ using similar considerations. Making use of Eq. $(A-28), P_{e}\left(\tilde{x}_{n}\right)$ is bounded by

$$
P_{e}\left(\tilde{x}_{n}\right) \geqslant e^{-d\left(\tilde{x}_{n}\right)} \phi\left(\sqrt{c\left(\tilde{x}_{n}\right)}\right)
$$

where $c\left(\tilde{x}_{n}\right)$ and $d\left(\tilde{x}_{n}\right)$ are obtained by substituting Eq. (19) into Eq. $(A-29)$ and $(A-30)$, and calculating these functions for $x_{n} \tilde{x}_{n}$. Following some straight forward algebra manipulations, one obtains $c\left(\tilde{x}_{n}\right)=\frac{w T}{2 \pi} \cdot \frac{1}{w \tilde{x}_{n} / 2} \int_{-w \tilde{x}_{n} / 4}^{w \tilde{x}_{n} / 4} \operatorname{SNR\cdot \operatorname {sin}^{2}\Omega d\Omega }$ $(\mathrm{C}-8)$ 
- $37-$

$$
d\left(\tilde{x}_{n}\right)=\frac{w T}{4 \pi} \frac{1}{w \tilde{x}_{n} / 2} \int_{-w \tilde{x}_{N / 4}}^{w \tilde{x}_{n} / 4}\left[S N R \cdot \sin ^{2} \Omega\right]^{2} d \Omega
$$

Using the inequality $\sin ^{2} \Omega \leqslant \Omega, c\left(\tilde{x}_{n}\right)$ and $d\left(\tilde{x}_{n}\right)$ can be bounded by

$$
\begin{aligned}
& c\left(\tilde{x}_{n}\right) \leq \tilde{c}^{2} \tilde{x}_{n}{ }^{2} \\
& d\left(\tilde{x}_{n}\right) \leq d^{H} \tilde{x}_{n}{ }^{4}
\end{aligned}
$$

$(C-11)$

where

$$
\begin{aligned}
& \tilde{c}^{2}=\frac{w^{3} T S N R}{48 \pi} \\
& \tilde{d}^{4}=\frac{W^{5} T S N R^{2}}{5 \cdot 2^{10} \cdot \pi}
\end{aligned}
$$

For future manipulations we note that

$$
(\tilde{c} / \tilde{d})^{4}=\frac{40}{9}(w T / 2 \pi)
$$

Substituting $C\left(\tilde{x}_{n}\right)$ and $d\left(\tilde{x}_{n}\right)$ by their upper bounds, one obtains

$$
P e^{\left(\tilde{x}_{n}\right)} \geqslant e^{-\tilde{J}^{\mu} \tilde{x}_{n}{ }^{4}} \phi\left(\tilde{c} \tilde{x}_{n}\right)
$$

Now since 
$-38-$

$$
\begin{aligned}
& G\left[(D-x) P_{e}(x)\right] \geqslant\left(D-\tilde{x}_{n}\right) P_{e}\left(\tilde{x}_{n}\right) \quad \tilde{x}_{n}-2 \pi / \omega_{0} \leqslant x \leqslant \tilde{x}_{n} \\
& \text { (C-16) } \\
& \text { and since the right hand side of Eq. }(C-15) \text { is a monotonically decreasing } \\
& \text { function, it immediately follows that } \\
& G\left[(D-x) P_{e}(x)\right] \geqslant\left(D-2 \pi / \omega_{0}-x\right) e^{-\tilde{d}-\left(x+2 \pi / \omega_{0}\right)^{4}} \phi\left[\tilde{c}\left(x+2 \pi / \omega_{0}\right)\right] \\
& (C-17) \\
& \text { Combining Es. (B-12), (C-17) and (B-11) (in the given order), } \\
& G\left[(D-x) P_{2}(x)\right] \quad \text { is tightly bounded by } \\
& (D-x) e^{-c^{14} x^{4}} \phi(c x) \\
& c \leqslant x<z_{1} \\
& \left.\xi[D-x) P_{e}(x)\right] \geqslant\{ \\
& \left(D-2 \pi / \omega_{0}-\tilde{d}^{4}\left(x+2 \pi / \omega_{0}\right)^{4}\right. \\
& \phi\left[\tilde{c}\left(x+2 \pi / \omega_{2}\right)\right] \quad \delta_{1} \leq x<z_{2}(c-18) \\
& (D-\pi / \omega-x) e^{2+b} \phi(\sqrt{2 b}) \\
& 3_{2} \leqslant x<D-2 \pi / w \\
& D-2 \pi / w \leq x \leq D
\end{aligned}
$$

where $z_{1}$ and $z_{1}$ are defined by

$$
\begin{aligned}
& c z_{1}=\tilde{c}\left(z_{1}+2 \pi / \omega_{0}\right) \\
& \tilde{c}\left(j_{2}+2 \pi / \omega_{0}\right)=\sqrt{2 b}
\end{aligned}
$$

Note that $z_{1}$ is approximately the point at which the first and second lines in Eq. (C-18) intersect, $z_{2}$ is approximately the point at which the second and third lines intersect. Substituting Eq. (C-18) into Eq. (C-1), one obtains the following lower bound 
- $39-$

$$
\begin{aligned}
\overline{\epsilon^{2}} \geqslant & \frac{1}{D} \int_{0}^{z_{1}} x(D-x) e^{-d^{4} x^{4}} \phi(c x) d x \\
& +\frac{1}{D} \int_{z_{1}}^{z_{2}} x\left(D-2 \pi / \omega_{0}-x\right) e^{-d^{4}\left(x+2 \pi / \omega_{0}\right)^{4}} \phi\left[\tilde{c}\left(x+2 \pi / \omega_{0}\right)\right] d x \\
& +\left[\frac{1}{D} \int^{D-2 \pi / w} x(D-2 \pi / w-x) d x\right] e^{a+b} \phi(\sqrt{2 b})
\end{aligned}
$$

$(C-21)$

Following some straight forward algebra manipulations, it can easily be shown that $z_{1} \ll 2 \pi / \omega_{0}$, and that $z_{2}<2 \pi / w$. Since we are assuming that $2 \pi / w \ll D$, the lower bound can be approximated, without incurring any significant errors, by

$$
\begin{aligned}
& \overline{\epsilon^{2}} \geqslant \sim \int_{0}^{\delta_{1}} x e^{-d^{4} x^{4}} \phi(c x) d x \text {. } \\
& +\int_{j^{2}}^{\xi^{2}} x e^{-\tilde{d}^{4}\left(x+2 \pi / \omega_{0}\right)^{4}} \phi\left[\tilde{c}\left(x+2 \pi / \omega_{0}\right)\right] d x+\frac{\partial^{2}}{b} e^{a+b} \phi(\sqrt{2 b}) \\
& =\frac{1}{c^{2}} \int_{0}^{1} \hat{d}^{\prime} x \cdot \exp \left\{-\frac{x^{4}}{(c / d)^{4}}\right\} \phi(x) d x \\
& +\frac{1}{\tilde{c}^{2}} \int_{\tilde{c}\left(z_{1}+2 \pi / \omega_{0}\right)}^{\tilde{c}\left(z_{2}+2 \pi / \omega_{0}\right)}\left(x-\tilde{c} \cdot 2 \pi / \omega_{0}\right) \exp \left\{-\frac{x^{4}}{(\tilde{c} / d)^{4}}\right\} \phi(x) d x+\frac{D^{2}}{b} e^{a+b} \phi(\sqrt{2 b}) \\
& =\frac{1}{c^{2}} \int_{0}^{\tau\left(z_{1}+2 \pi / \omega_{0}\right)} x \cdot \exp \left\{-\frac{x^{4}}{z(w / 2 \pi)}\right\} \phi(x) d x \\
& +\frac{1}{\tilde{c}^{2}} \int\left(x-\tilde{c} 2 \pi / \omega_{0}\right) \exp \left\{-\frac{9 x^{4}}{H_{0}(W T / 2 \pi)}\right\} d(x) c \psi+\frac{\partial^{2}}{b} e^{a+b} \phi(\sqrt{2 b}) \\
& \tilde{c}\left(z_{1}+2 \pi / \omega_{0}\right) \\
& \approx \frac{1}{c^{2}} \int_{0}^{\tilde{c} 2 \pi / w \cdot} x \cdot \exp \left\{-\frac{x^{4}}{g(w T / 2 \pi)}\right\} \phi(x) d x \\
& +\frac{1}{\tilde{c}^{2}} \int_{\tilde{c}_{2} \pi / \omega}^{\sqrt{2 b}}\left(x-\tilde{c} 2 \pi / \omega_{0}\right) \cdot \exp \left\{\cdot \frac{9 x^{4}}{40(\omega t / 2 \pi)}\right\} \phi(x) d t+\frac{D^{2}}{6} e^{\alpha+b} \phi(\sqrt{2 b})
\end{aligned}
$$


where in the transition from the second version of Eq. (C-22) to its third version we have substituted Eqs. $(C-4),(C-14),(C-19)$ and $(C-20)$. Substituting Eqs. (C-2), (C-12), (C-5) and (C-6) into Eq. (C-22), one can now generate m.s.e. predictions for any pre-specified SNR. We further note that the second and third terms in Eq. (C-22) contribute significantly to the sum only when SNR $<<1$. In that region, $a$ and $b$ can be approximated, without incurring any significant errors, by

$$
a \simeq-\frac{w T}{\pi} \cdot \frac{S N R}{4}
$$

$$
0 \approx \frac{W T}{2 \pi} \cdot \frac{S A R}{2}
$$

With these considerations, the lower bound assumes the form

$$
\frac{\pi}{6} \frac{\omega}{\omega}, \bar{R}
$$

$E^{2} \geqslant \ldots \int_{0} x \cdot \operatorname{sxp}\left\{-\frac{x^{4}}{q(w+2 \pi)}\right\} \phi(x) d x$

$$
\begin{aligned}
& +\frac{1}{\left(W^{2} / 24\right) R} \int^{\hat{R}} x \cdot \exp \left\{-\frac{9 x^{4}}{40(W T / 2 \pi)}\right\} \phi(x) d x+\frac{D^{2}}{6} \phi(\sqrt{R}) \\
& \sqrt{\frac{\pi^{2}}{6}} \frac{\omega}{\omega_{0}} \sqrt{R}
\end{aligned}
$$

where $R$ is defined as the product of $W T / 2 \pi$ by SNR (the so-called post-integration SNR).

We shall now examine few limiting cases: If $R \ll 1$, the third term on the right hand side of Eq. (C-25) becomes the dominant term in the sum and the lower bound approaches 
$\overline{\epsilon^{2}} \geqslant \sim \frac{D^{2}}{6} \phi(\sqrt{R}) \longrightarrow \frac{D^{2}}{12}$

If $1 \ll R \ll\left(w_{0} / w\right)^{2}$, the second term becomes the dominant term in the sum and the lower bound approaches

$$
\overline{\epsilon^{2}} \geqslant \sim \frac{1}{\left(w^{2} / 24\right) R} \int_{\sqrt{\frac{\pi^{2}}{6}} \frac{w}{\omega_{0}} \sqrt{R}}^{\sqrt{R}} x \cdot \exp \left\{-\frac{9 x^{4}}{4 n(w T / 2 \pi)}\right\} \phi(x) d t \quad \underset{(\omega T / 2 \pi)>1}{\longrightarrow}
$$$$
\frac{1}{\left(w^{2} \sqrt{12) R}\right.} \phi\left(\sqrt{\frac{\pi^{2}}{6}} \frac{\omega}{\omega_{0}} \sqrt{R}\right) \approx \frac{1}{\left(w_{0}^{2} / 6\right.}
$$

Finally, if $R>(\omega . / w)^{2}$, the first term in the sum becomes the dominant one, and the lower bound approaches

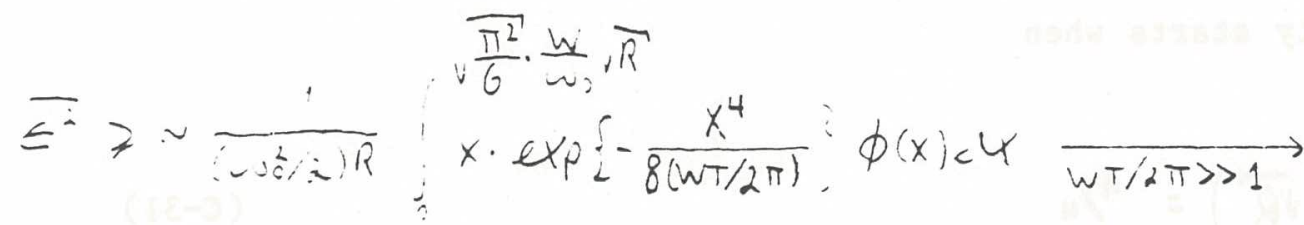

$$
\begin{aligned}
& \frac{1}{2 \omega_{0}^{2} R}\left[1-20\left(\sqrt{\frac{E^{2}}{6}} \frac{w}{\omega_{0}} \cdot \bar{R}\right)\right] \approx \frac{1}{2 \omega_{0}^{2} R}
\end{aligned}
$$

One immediately identifies Eqs. $(\mathrm{C}-26),(\mathrm{C}-27)$ and $(\mathrm{C}-28)$ with the first, third and fifth lines of Eq. (22) respectively. The transitions from the first line of Eq. (22) to its third line essentially starts when 


$$
\phi(\sqrt{R})=\frac{1}{4}
$$

and is essentially completed when

$$
\frac{D^{2}}{6} \phi(\sqrt{R})=\frac{1}{\left(W^{2} / 6\right) R}
$$

where in the transition region the lower bound varies essentially as $\phi(\mathbb{R})$. Denoting by $R=x$ the solution to Eq. $(C-29)$ and using the similarity between Eq. (C-29) and Eq. (B-28), one immediately obtains Eq. (26). Denoting by $R: \delta$ the solution to Eq. (C-30) and using the similarity between Eq. $(C-30)$ and Eq. $(B-29)$, one immediately obtains Eq. (27).

The transition from the third line of Eq. (22) to its fifth line essentially starts when

$$
\left.+\sqrt{\frac{-2}{6}} \frac{\omega}{L_{i j}} \sqrt{R}\right)=1 / 4
$$

and is essentially completed when

$$
\left.\frac{1}{\left(w^{2} / 12\right) R} \phi \sqrt{\frac{\pi^{2}}{6}} \frac{w}{\omega_{0}} \sqrt{R}\right)=\frac{1}{2 \omega_{0}^{2} R}
$$

where in the transition region the lower bound varies essentially as $\phi\left(\sqrt{\frac{\pi^{2}}{G}} \frac{\omega}{\omega_{0}} \sqrt{R}\right)$. Denoting by $R=\mu$ and $R=\eta$ the solutions to Eqs. $(C-31)$ and $(C-32)$ and following some straight forward algebra manipulations, one immediately obtains Eq. (24) and (25), respectively. 


\section{References}

1. V.H. MacDonald and P.M. Schultheiss, "Optimum Passive Bearing Estimation in a Spatially Incoherent Noise Environment", J. Acoust. Soc. Am. 46, pp. 37-43, 1969.

2. W.R. Hahn, "Optimum Signal Processing for Passive Sonar Kange and Bearing Estimation", J. Acoust. Soc. Am. 58 (1), Pp. 201-207.

3. G.C. Carter, "Time Delay Estimation for Passive Sonar Signal Processing", IEEE Trans. Acoust. Speech, Signal Processing, Vol. ASSP-29, pp. 463-470, June 1981.

4. Y.T. Chan, R.V. Hattin and J.B. Plant, "The Least Squares Estimation of Time Delay and Its Use in Signal Detection", IEEE Trans. Acoust. Speech, Signal Processing, Vol. ASSP-26, No. 3, FP. 217-222, 1978.

5. W.R. Hahn and S.A. Tretter, "Optimum Processing for Delay-Vector Estimation in Passive Signal Arrays", IEEE Trans. on Information Theory, Vol. IT-19, No. 5, pp. 608-614, 1973.

6. B.V. Hamon and E.J. Hannan, "Spectral Estimation of Time Delay for Dispersive and Non-Dispersive Systems", J. Appl. Statis 23 (2), pp. $134-142,1974$.

7. C.H. Knapp and G.C. Carter, "The Generalized Correlation Method for Estimation of Time Delay", IEEE Irans. Acoust. Speech, Signal Processing, Vol. ASSP-24, No. 4, PP. 320-327, 1976.

8. A. Weiss and E. Weinstein, "Composite Bound on the Attainable Mean-Square Error in Passive Time-Delay Estimation from Ambiguity Prone Signal", IEEE Trans. on Information Theory, Vo1. IT-28, No. 6, pp. 977-979, November 1982 .

9. A.J. Weiss and E. Weinstein, "Fundamental Limitations in Passive Time Delay Estimation - Part I: Narrowband Systems", IEEE Trans. Acoust. Speech, Signal Processing, Vol. ASSP-31, pp. 472-486, April 1983.

10. J.P. Ianniello, E. Weinstein and A. Weiss, "Comparison of the Ziv-Zakai Lower Bound on Iime Delay Estimation with Correlator Performance", Proceedings of the IEEE International Conference on Acoustics, Speech and Signal Processing (ICASSP 83), April 1983.

11. S.K. Chow and P.M. Schultheiss, "Delay Estimation using Narrowband Processes", IEEE Trans. Acoust. Speech, Signal Processing, Vol. ASSP-29, No. 3, PP. 478-484, June 1981.

12. I.S. Gradshteyn, I.M. Ryzhik, "Tables of Integrals, Series and Products", Academic Press, 1980. 


\section{Figure Captions}

Fig. 1. Typical Narrowband Signal Cross Correlation

Fig. 2. Typical Baseband Signal Cross Correlation

Fig. 3. Typical Bandpass Signal Cross Correlation

Fig. 4. Composite Bound on $\overline{\epsilon^{2}}$ - Baseband Systems

Fig. 5. $\beta$ vs. $W D / 2 \pi$

Fig. 6. $\overline{\epsilon^{2}}$ VS. Post Integration SNR for Easeband Signals.

Fig. 7. Composite Bound on $\overline{\epsilon^{2}}$ - Bandpass Systems

Fig. 8. Normalized m.s.e. vs. Post Integration SNR for $\left(v_{,}, h_{=}=0\right)$

Fig. 9. Normalized m.s.e. vs. Post Integration SNR for $\omega_{2} / w=20$

Fig. 10. Normalized m.s.e. vs. Post Integration SNR for $\omega_{0} / w=5$

Fig. 11. Composite Eound on $\overline{\epsilon^{2}}$ - Narrowband Systems.

Fig. 12. $P_{e}(x)$ and $C\left[(D-x) P_{e}(x)\right]$ for Typical Easeband Case. 


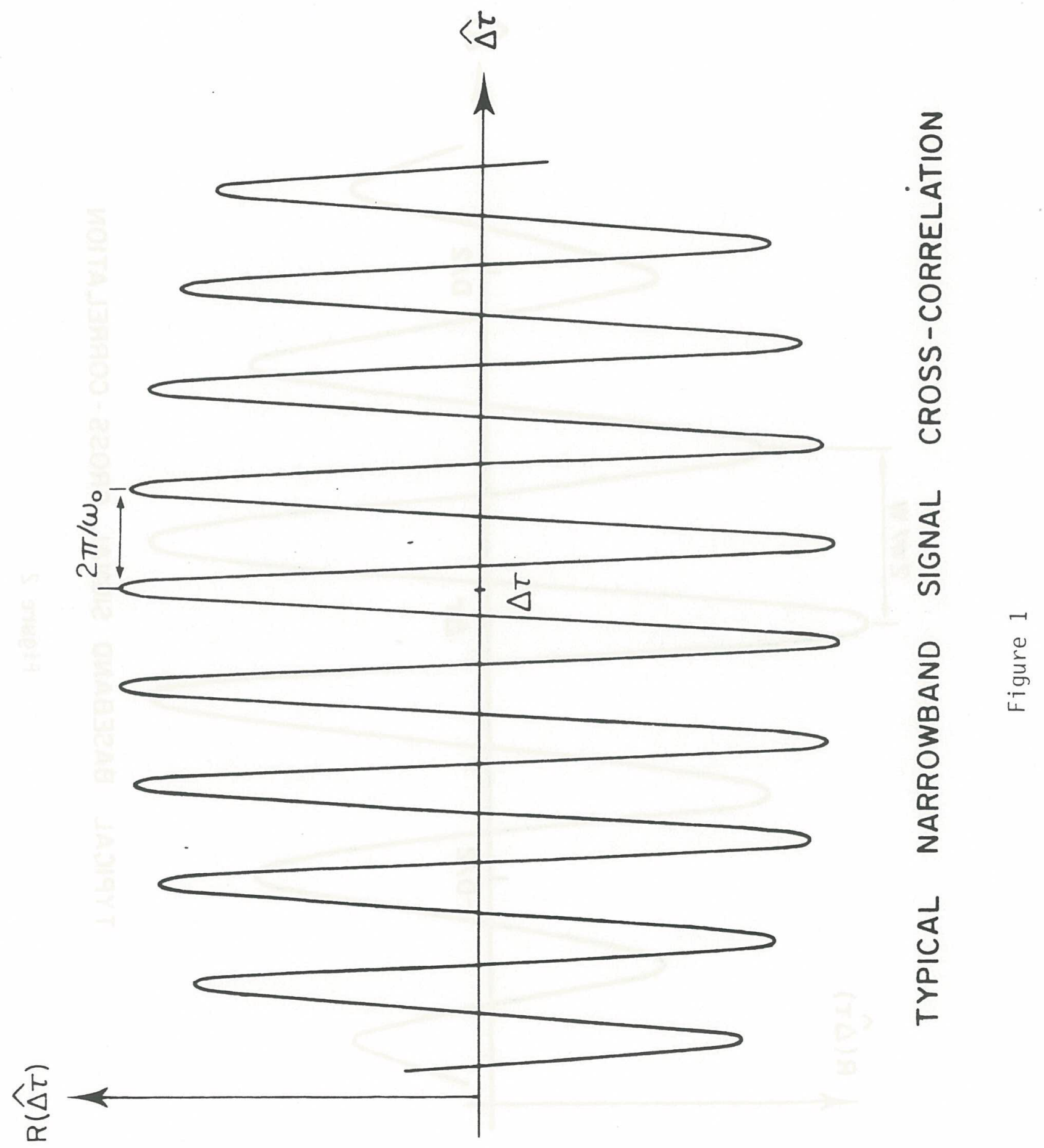




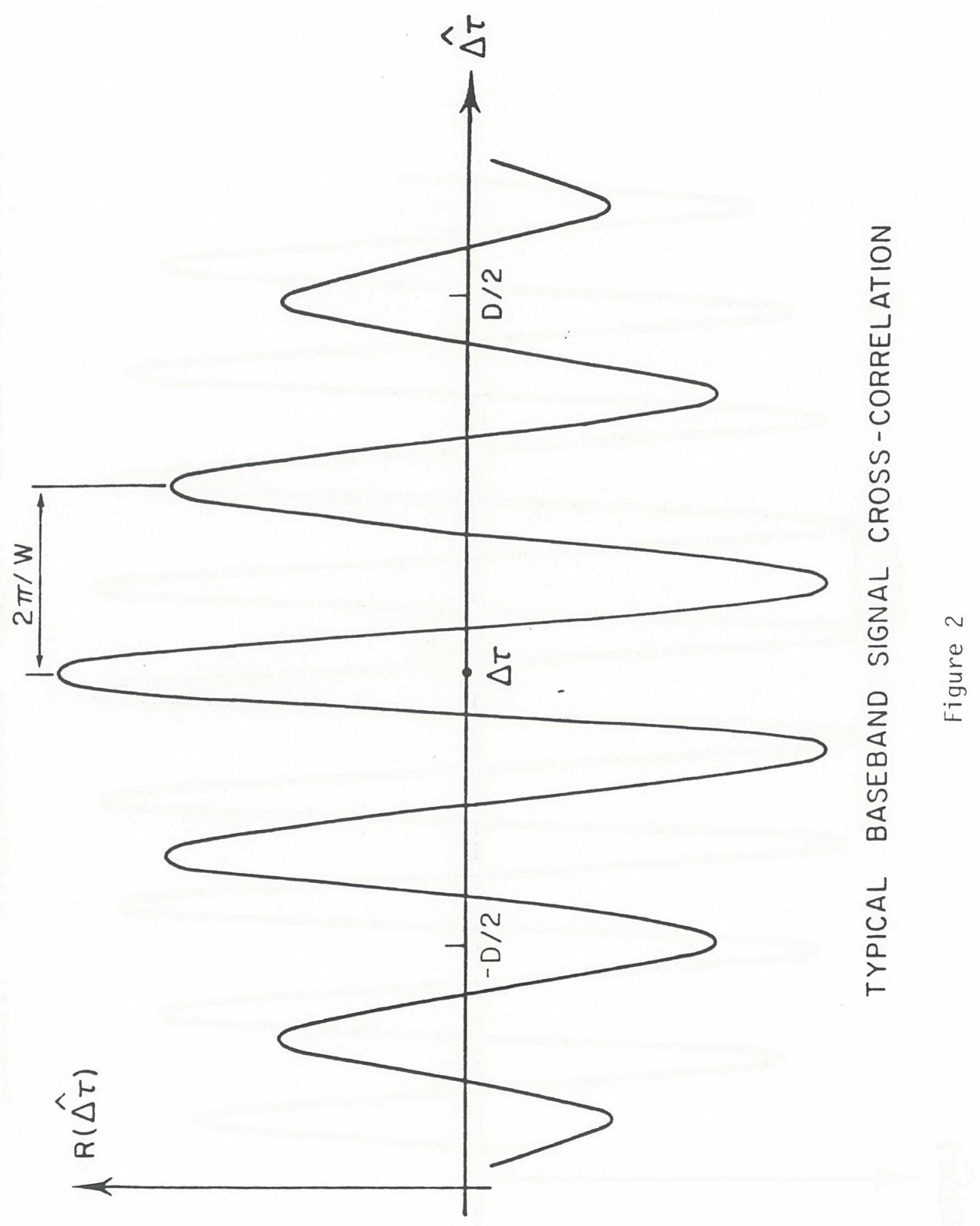




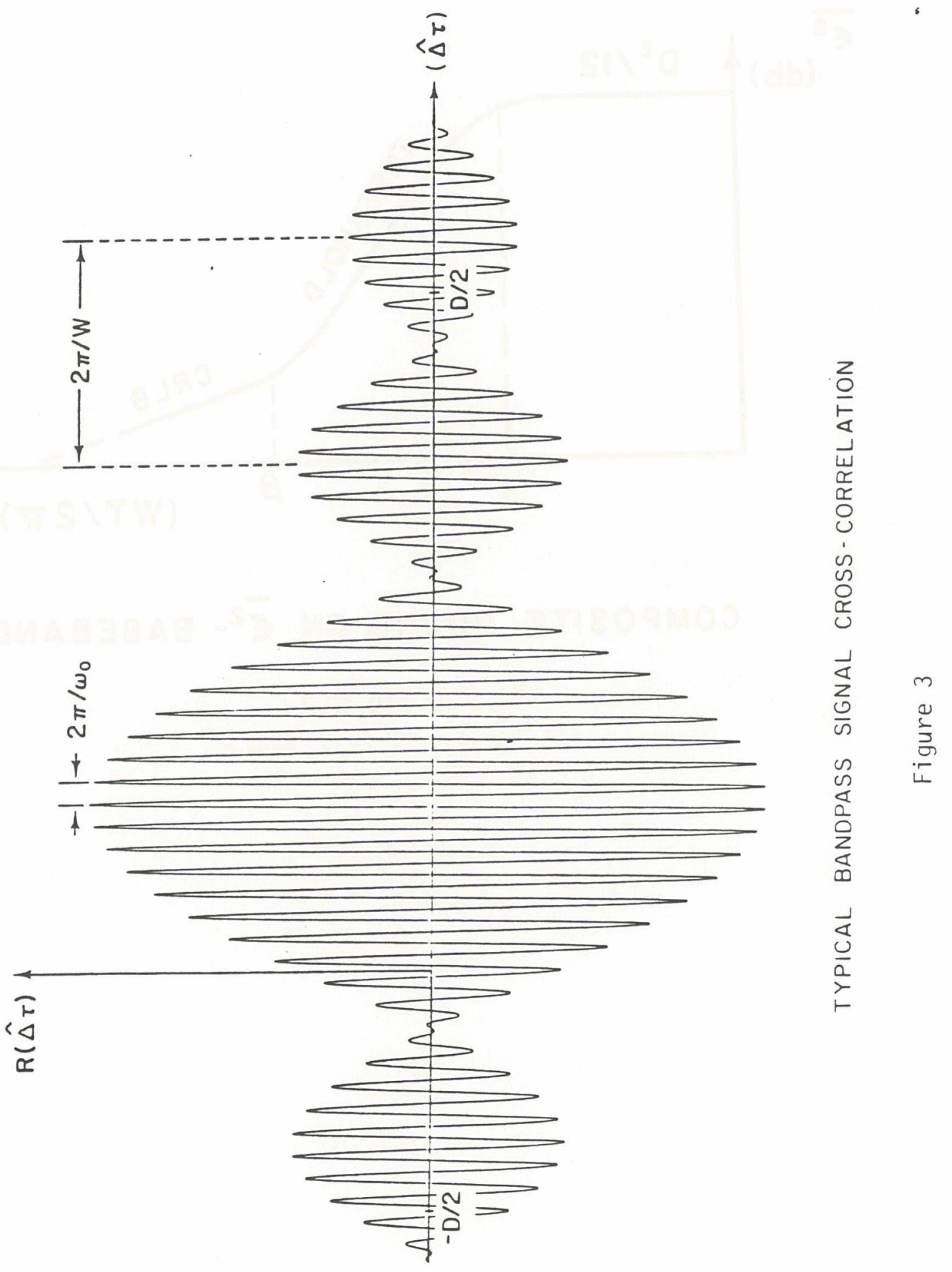




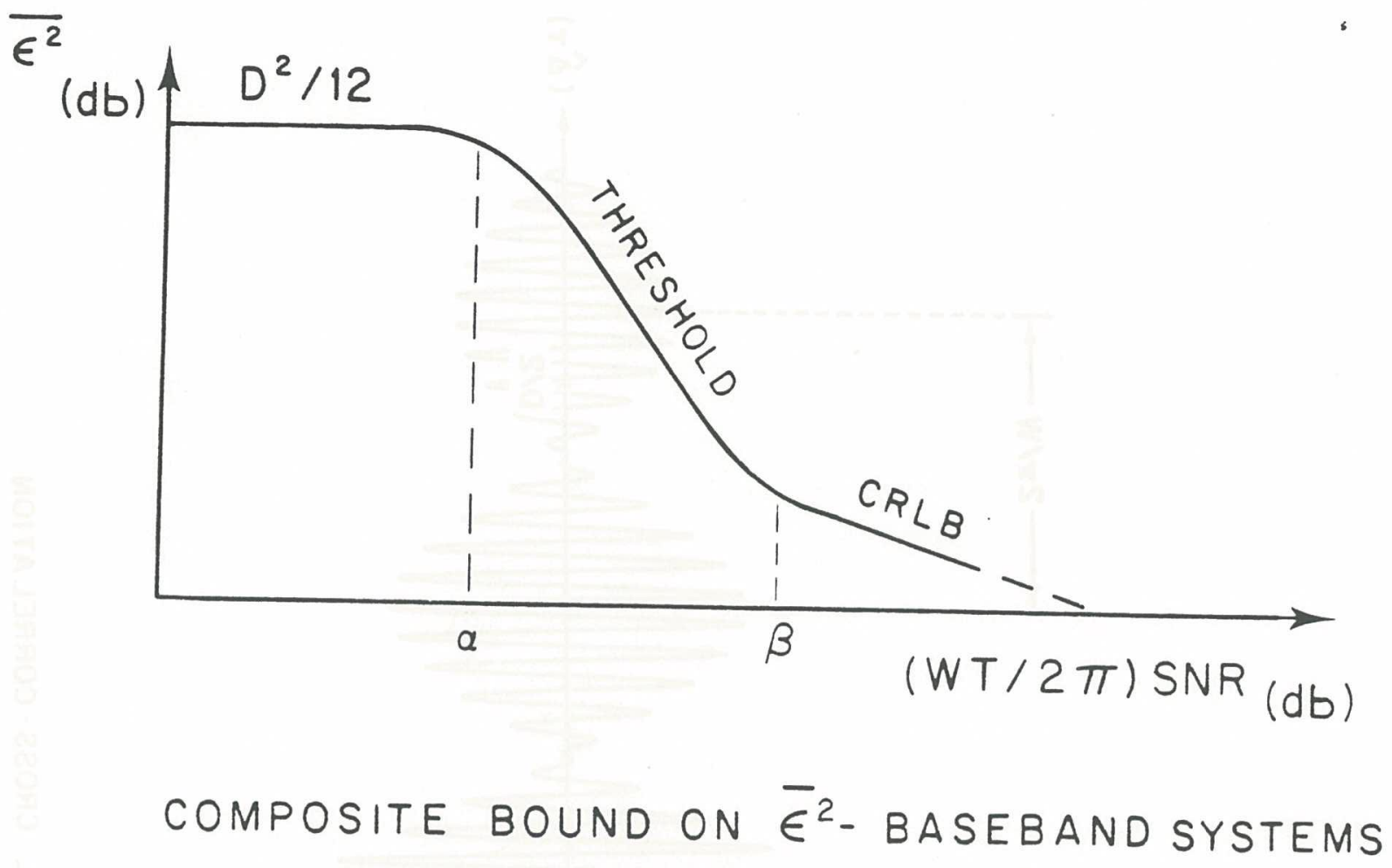

Figure 4 


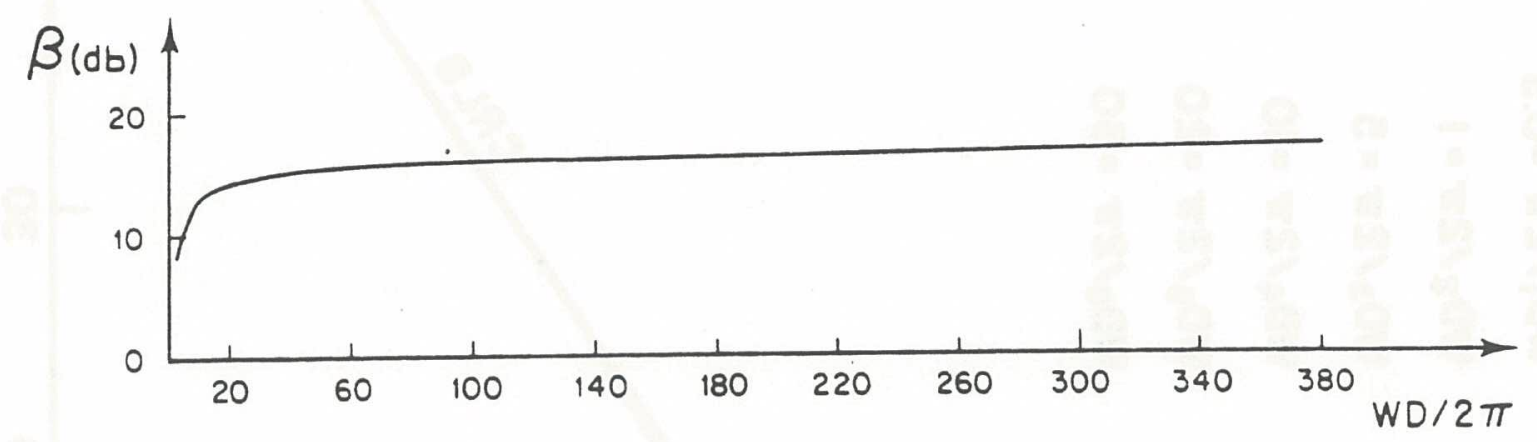

\begin{tabular}{c|c|c}
$W D / 2 \pi$ & $\beta$ & $\beta(d b)$ \\
\cline { 2 - 3 } 3 & 7.38 & 8.68 \\
10 & 19.33 & 12.86 \\
20 & 25.44 & 14.06 \\
60 & 34.98 & 15.44 \\
100 & 39.34 & 15.95 \\
140 & 42.17 & 16.25 \\
180 & 44.30 & 16.46 \\
220 & 45.91 & 16.62 \\
260 & 47.31 & 16.75 \\
300 & 48.60 & 16.87 \\
340 & 49.56 & 16.95 \\
380 & 50.53 & 17.04
\end{tabular}

$\beta$ VS. WD $/ 2 \pi$

Figure 5 


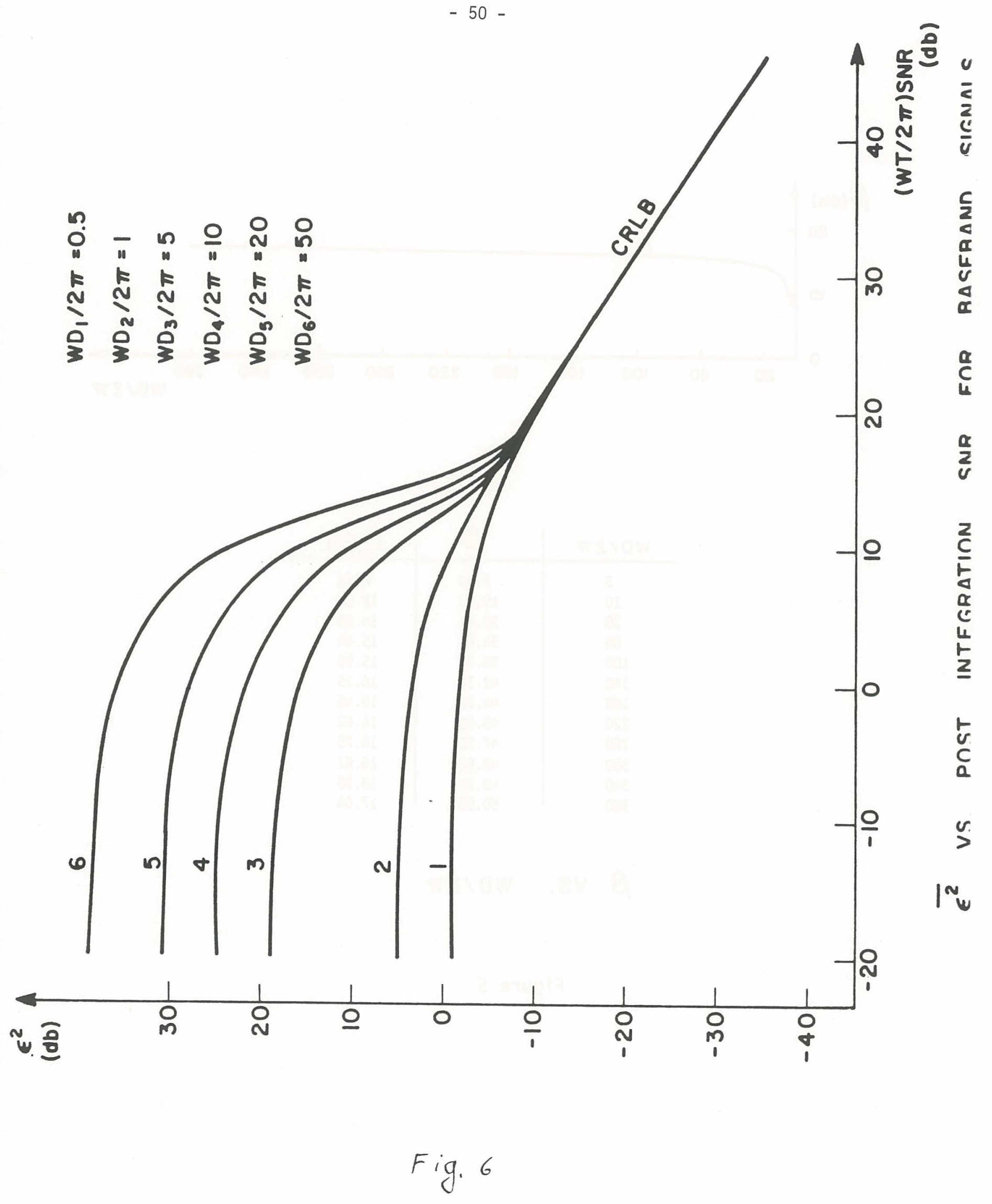




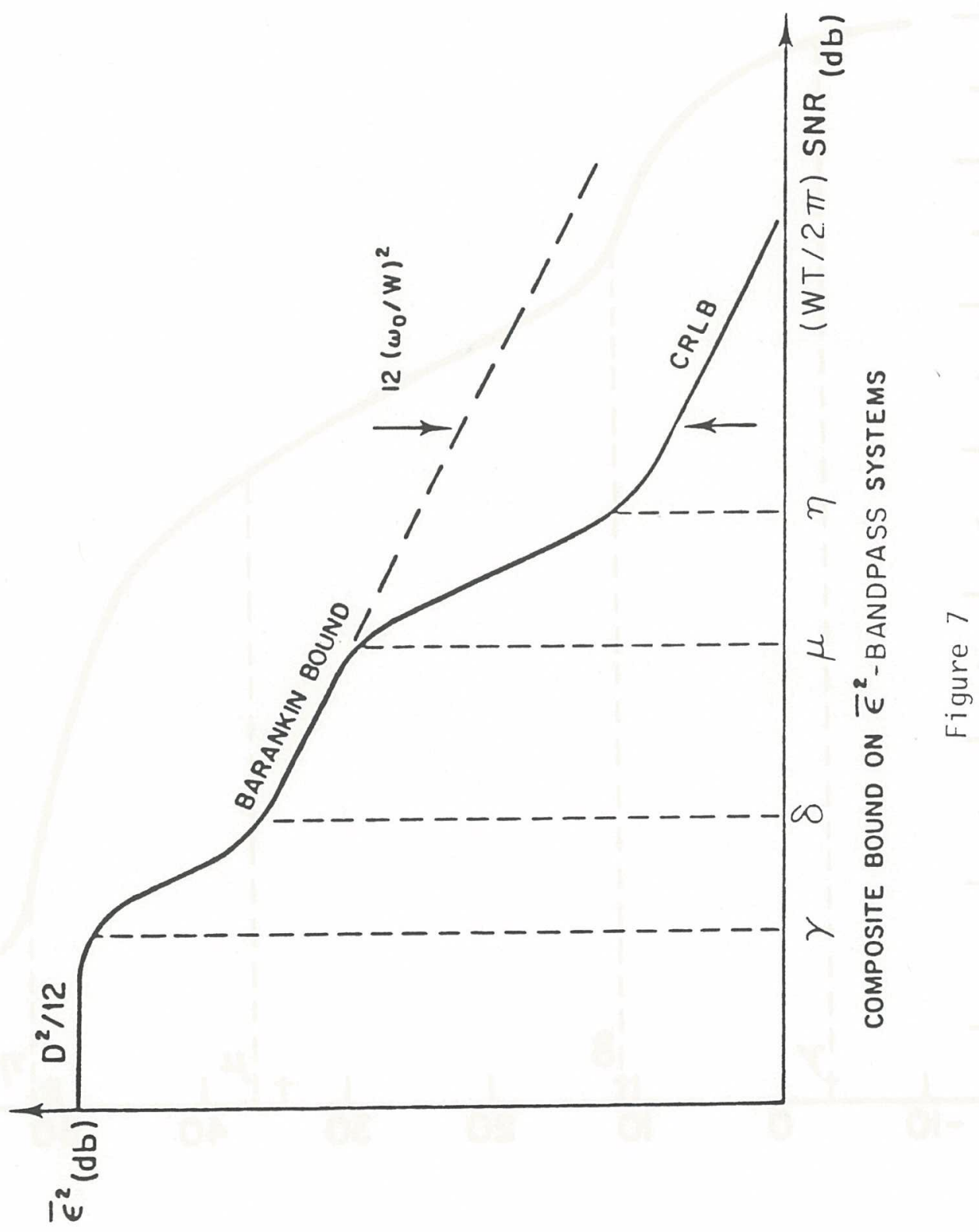


$\overline{\epsilon^{2} / 1 D^{2} / 12}$

(db)
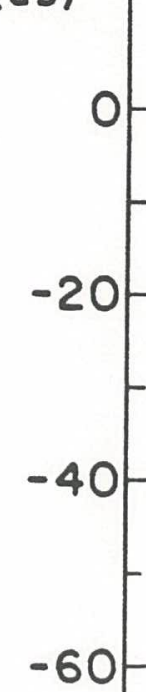

$-80$

$-100$

$-120$

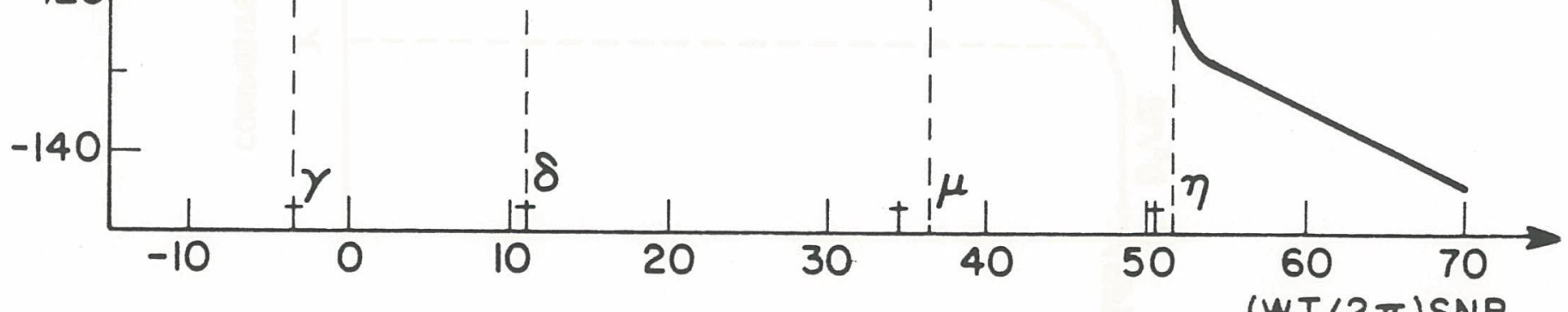

(WT/2 $\pi$ )SNR

(db)

NORMALIZED M.S.E. VS. POST INTEGRATION SNR FOR $\omega_{0} / W=100$

Figure 8 


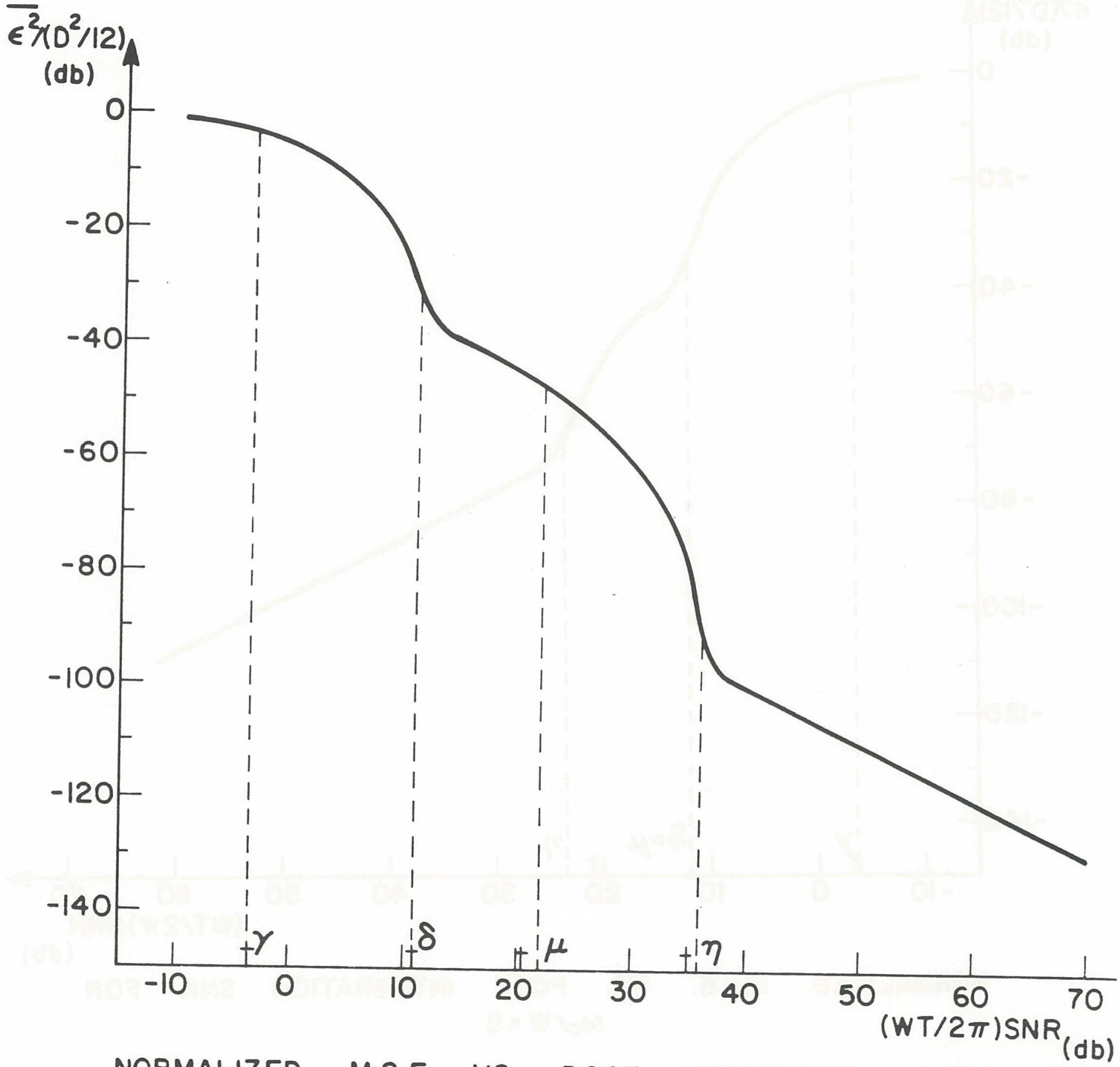

NORMALIZED M.S.E. VS. POST INTEGRATION SNR FOR $\omega_{0} / w=20$

Figure 9 


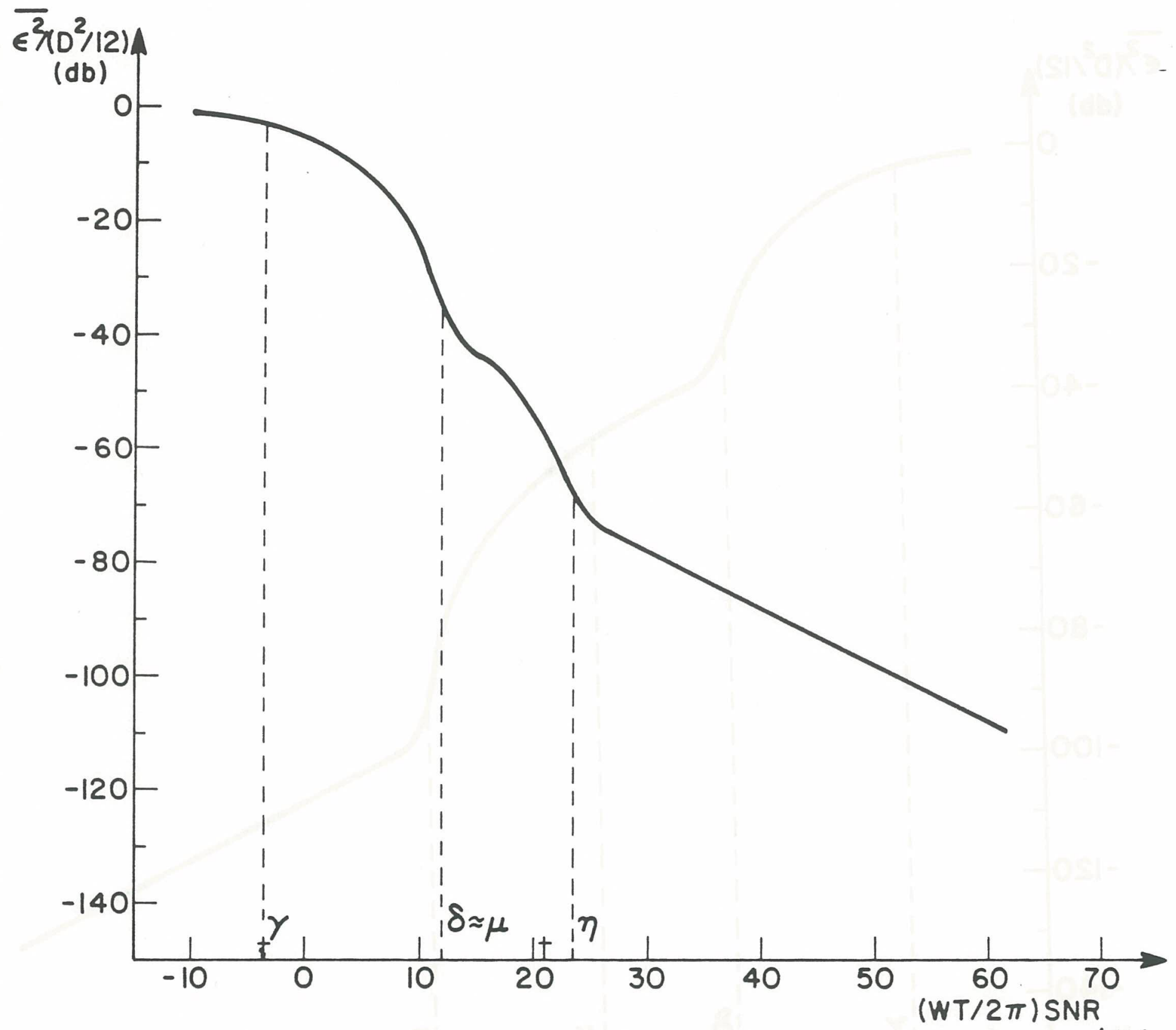

NORMALIZED M.S.E. VS. POST INTEGRATION SNR FOR $\omega_{0} / W=5$

Figure 10 


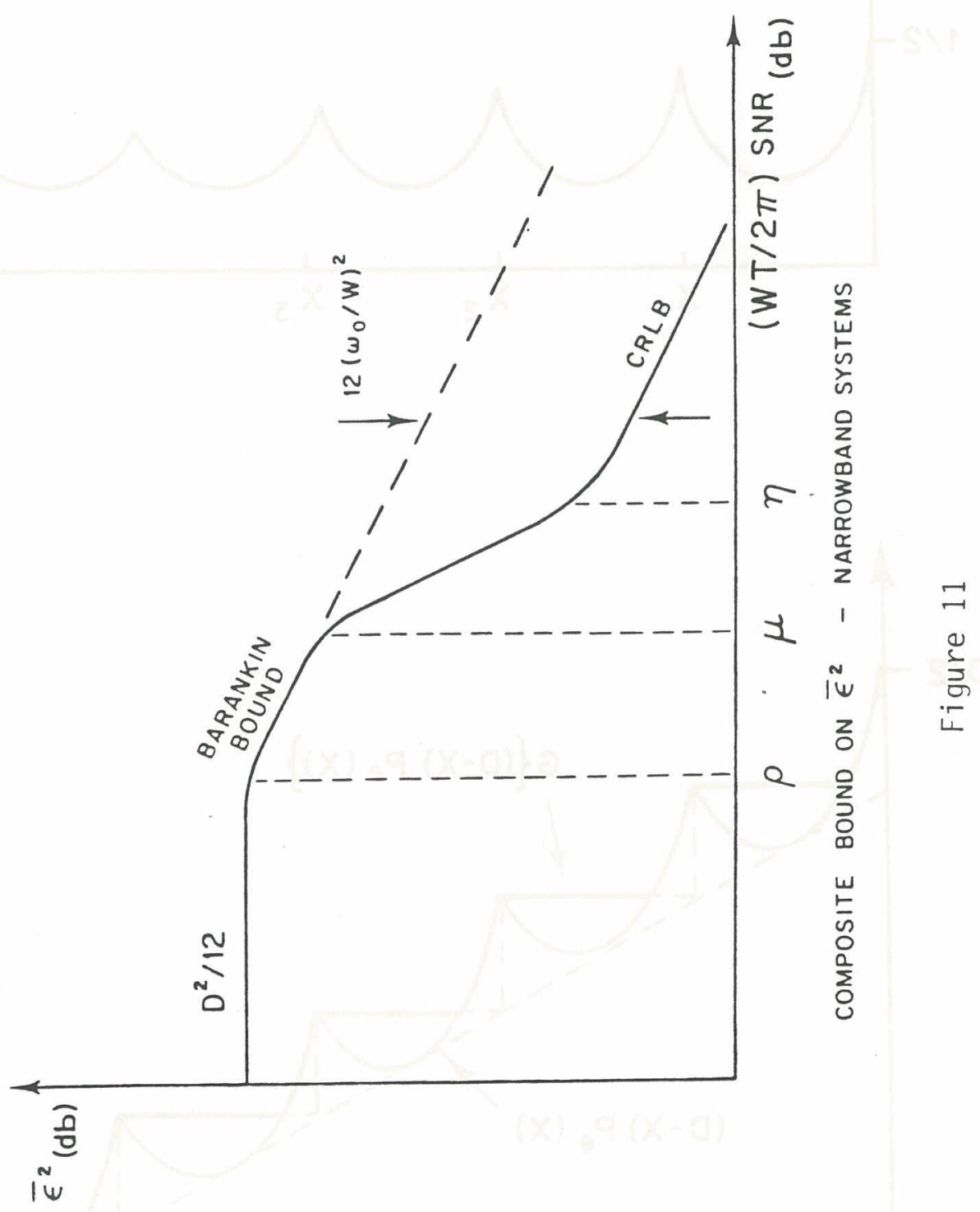



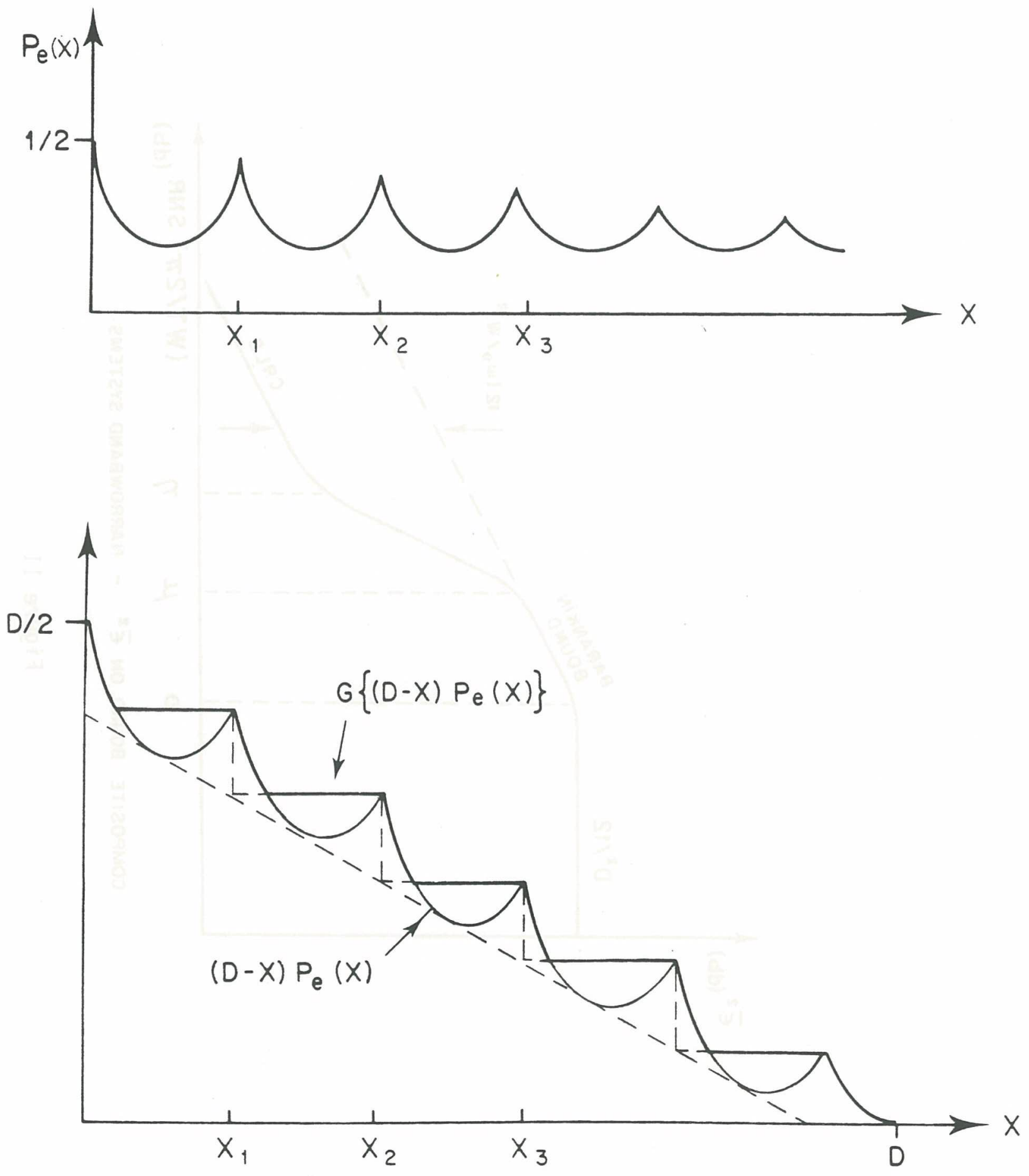

Figure 12 


\section{DOCUMENT LIBRARY}

August 3, 1984

DISTRIBUTION LIST FOR TECHNICAL REPORT EXCHANGE

Institute of Marine Sciences Library

University of Alaska

O'Neill Building

905 Koyukuk Ave. North

Fairbanks, AK

Attn: Stella Sanchez-Wade

Documents Section

Scripps Institution of Oceanography

Library, Mail Code C-075C

La Jolla, CA 92093

Hancock Library of Biology \& Oceanography

Alan Hancock Laboratory

University of Southern California

Los Angeles, CA 90007

Gifts \& Exchanges

Library

Bedford Institute of Oceanography

P.O. Box 1006

Dartmouth, NS, B2Y 4A2, CANADA

Office of the International

Ice Patrol

c/o Coast Guard R\&D Center

Avery Point

Groton, CT 06340

Library

Physical Oceanographic Laboratory

Nova University

8000 N. Ocean Drive

Dania, FL 33304

NOAA/EDIS Miami Library Center 4301 Rickenbacker Causeway

Miami, FL 33149

\section{Library}

Skidaway Institute of Oceanography

P.O. Box 13687

Savannah, GA 31406

Institute of Geophysics

University of Hawaii

Library Room 252

2525 Correa Road

Honolulu, HI 96822
Library

Chesapeake Bay Institute

4800 Atwell Road

Shady Side, MD 20876

MIT Libraries

Serial Journal Room 14E-210

Cambridge, MA 02139

Director, Ralph M. Parsons Laboratory

Room 48-311

MIT

Cambridge, MA 02139

Marine Resources Information Center

Bldg. E38-320

MIT

Cambridge, MA 02139

Library

Lamont-Doherty Geological Observatory

Colombia University

Palisades, NY 10964

Library

Serials Department

Oregon State University

Corvallis, OR 97331

Pell Marine Science Library

University of Rhode Island

Narragansett Bay Campus

Narragansett, RI 02882

Working Collection

Texas A\&M University

Dept. of Oceanography

College Station, TX 77843

Library

Virginia Institute of Marine Science

Gloucester Point, VA 23062

Fisheries-Oceanography Library

151 Oceanography Teaching Bldg.

University of Washington

Seattle, WA 98195

Library

R.S.M.A.S.

University of Miami

4600 Rickenbacker Causeway

Miami, FL 33149 



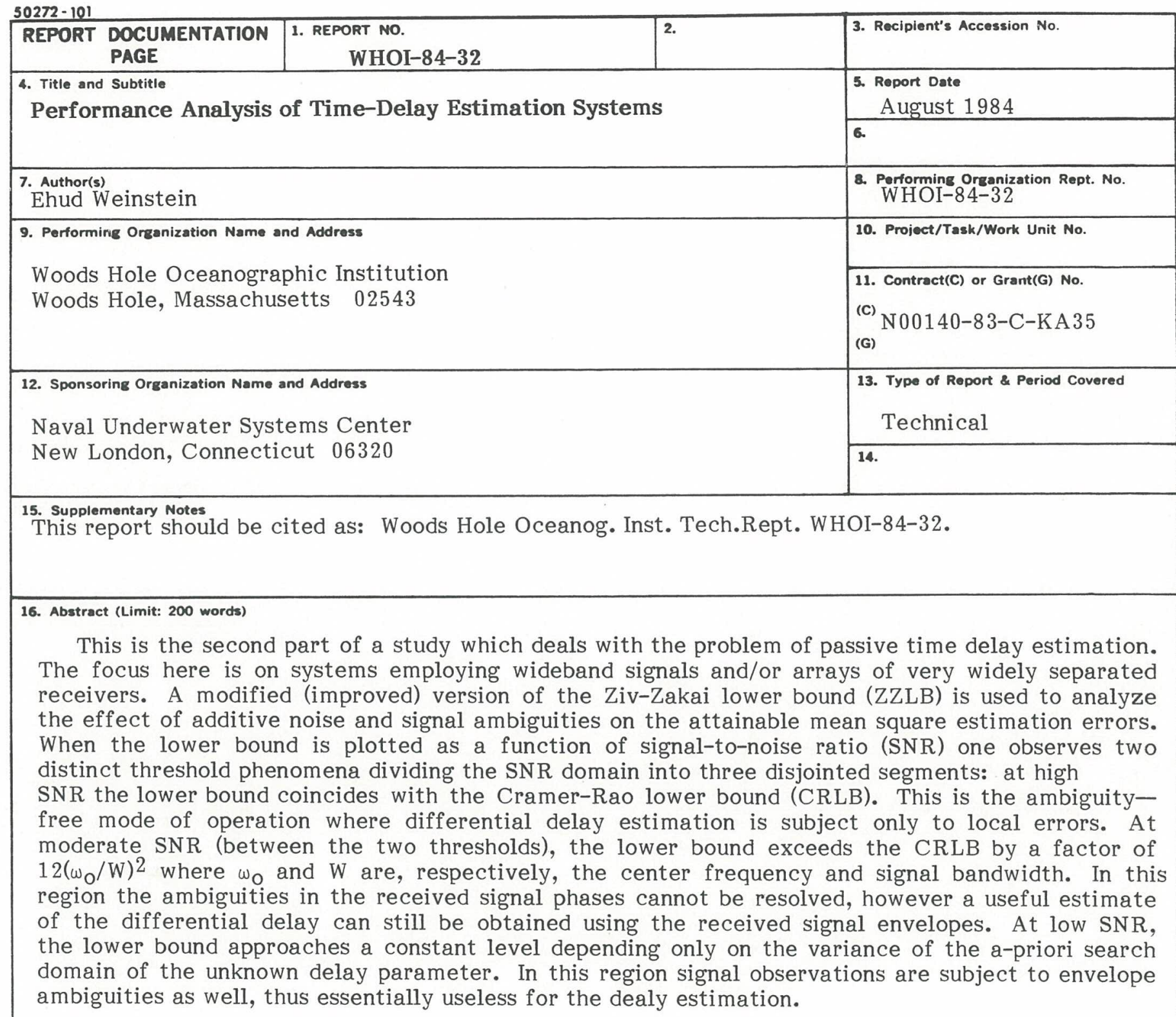

17. Document Analysis a. Descriptors

1. Time Delay Estimation

2. Envelop and Phase Ambiguities

3. Threshold Effects

b. Identifiers/Open-Ended Terms

c. COSATI FieId/Group

18. Availability Statemen:

Approved for publication; distribution unlimited. 
